\title{
Cholesterol catalyses $A \beta 42$ aggregation through \\ a heterogeneous nucleation pathway in the presence of lipid membranes
}

\begin{abstract}
Johnny Habchi ${ }^{1 *}$, Sean Chia ${ }^{1 *}$, Céline Galvagnion ${ }^{1 \#^{*}}$, Thomas C. T. Michaels ${ }^{1}$, Mathias M. J. Bellaiche ${ }^{1,2}$, Francesco Simone Ruggeri ${ }^{1}$, Michele Sanguanini ${ }^{1}$, Ilaria Idini ${ }^{4}$, Janet R. Kumita ${ }^{1}$, Emma Sparr ${ }^{3}$, Sara Linse ${ }^{4}$,

Christopher M. Dobson ${ }^{1}$, Tuomas P. J. Knowles ${ }^{1,5}$, Michele Vendruscolo ${ }^{1+}$
\end{abstract}

${ }^{1}$ Department of Chemistry, University of Cambridge, Cambridge CB2 1EW, UK

${ }^{2}$ Laboratory of Chemical Physics, National Institute of Diabetes and Digestive and Kidney Diseases, National Institutes of Health, Bethesda, MD 20892, USA

${ }^{3}$ Division of Physical Chemistry, Department of Chemistry, Lund University, P.O. Box 124, 22100, Lund, Sweden

${ }^{4}$ Department of Biochemistry \& Structural Biology, Center for Molecular Protein

Science, Lund University, P.O. Box 124, 22100 Lund, Sweden

${ }^{5}$ Department of Physics, Cavendish Laboratory, $19 \mathrm{~J} J$ Thomson Avenue, Cambridge CB3 OHE, UK

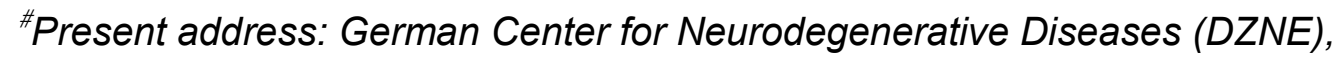
Sigmund-Freud-Strasse 27, D-53127, Bonn, Germany

*Equal contributions

+Corresponding author: mv245@cam.ac.uk

\section{Keywords}

Alzheimer's disease, amyloid- $\beta$ peptide; protein misfolding; protein aggregation; chemical kinetics; cholesterol; lipid vesicles; amyloid; heterogeneous nucleation

\section{Abbreviations}

$A D$, Alzheimer's disease; $A \beta$, amyloid- $\beta$ peptide; $A \beta 42$, 42-residue form of $A \beta$; ThT, thioflavin T; DMPC, 1,2-dimyristoyl-sn-glycero-3-phosphocholine. 
Alzheimer's disease (AD) is a neurodegenerative disorder associated with the aberrant aggregation of the amyloid- $\beta$ peptide $(A \beta)$. Although increasing evidence implicates cholesterol in AD pathogenesis, the detailed mechanistic link between this lipid molecule and the disease process remains to be fully established. To address this problem, we adopt a kinetics-based strategy that reveals a specific catalytic role of cholesterol in the aggregation of $A \beta 42$ (the 42-residue form of $A \beta$ ). More specifically, we demonstrate that lipid membranes containing cholesterol promote $A \beta 42$ aggregation by enhancing by up to 20 fold its primary nucleation rate through a heterogeneous nucleation pathway. We further show that this process occurs as a result of the cooperativity in the interaction of multiple cholesterol molecules with $A \beta 42$. These results identify a specific microscopic pathway by which cholesterol dramatically enhances the onset of $A \beta 42$ aggregation, thereby rationalising the link between $A D$ and increased levels of cholesterol in cell membranes.

Over 40 million people currently suffer from $A D$ worldwide, and as the global population ages, this number could approach 140 million by $2050^{1}$. The deposition of $A \beta$ into insoluble aggregates in brain tissue represents the molecular signature of the disease, with $A \beta 42$, the 42-residue form of $A \beta$, being the major component of the deposits $^{2-5}$. Therefore, understanding at the molecular level the effects of intrinsic and extrinsic factors on the aggregation process of $A \beta 42$ is vital for developing effective therapeutic strategies aimed at inhibiting its self-assembly ${ }^{6,7}$.

Among such factors, the disruption of lipid homeostasis in the brain is strongly associated with $A D$ pathogenesis ${ }^{8,9}$. Lipids are found ubiquitously within the amyloid deposits formed by $A \beta 42$ aggregates $^{10}$. It has also been suggested that the comparative analysis of the lipid composition of the plasma and cerebrospinal fluid (CSF) of AD patients and healthy controls could lead to the identification of effective disease biomarkers and prognostic indicators of $A D$ therapies ${ }^{9,10}$.

A critical role of lipids in $A D$ is consistent with the fact that the $\varepsilon 4$ allele of the apoliprotein $\mathrm{E}$ gene $(A P O E)$ is the greatest currently known genetic risk factor for late-onset $A D^{11,12}$. Apoliprotein $E$ is a crucial regulator of cholesterol metabolism in 
the brain and of triglyceride metabolism throughout the body ${ }^{13}$, and studies have shown that $A \beta$ deposits fail to form in $A P O E$ knockout mice ${ }^{14}$. Therefore, much attention has been devoted to the link between $A D$ and cholesterol. Recent studies have suggested that plasma cholesterol levels are about $10 \%$ higher in $A D$ patients when compared to healthy individuals ${ }^{15}$, and that cholesterol accumulates in senile plaques of $A D$ patients and in mouse models of $A D^{10,16}$. Furthermore, it has also been suggested that statins, which are used to prevent cardiovascular diseases by lowering cholesterol levels, could also potentially reduce the risk of $A D^{17}$. Brain cholesterol, which exists primarily $(>99.5 \%$ ) in a non-esterified state, is largely formed in the myelin sheaths and cellular membranes of glial cells and neurons ${ }^{18}$, represents about $25 \%$ of the total amount of cholesterol in the human body, and is important for neuronal development, synaptic plasticity, and brain function. The impairment of cholesterol homeostasis could therefore be an important factor in AD pathogenesis ${ }^{19}$.

The mechanistic processes underlying the association of cholesterol with AD remain, however, to be fully established ${ }^{20,21}$. Evidence for a possible causative role is provided by recent studies showing that modulating cholesterol levels can regulate $A \beta$ aggregation ${ }^{34,35}$. For example, depleting cholesterol in hippocampal neurons in $A D$ animal models has been found to reduce $A \beta 42$ aggregate levels, while increasing it leads to their increase ${ }^{22}$. Additionally, lipid rafts, which are domains in cellular membranes enriched in cholesterol and sphingolipids, have been implicated in the processing of the amyloid precursor protein (APP) through which $A \beta$ is generated $^{9,10,23}$. Moreover, the region of residues 22-35 in the $A \beta$ sequence has been identified as a potential binding site of cholesterol ${ }^{21}$.

Although an interaction between $\mathrm{A} \beta 42$ and cholesterol has already been reported ${ }^{24}$, the mechanisms by which it occurs and affects $A \beta 42$ aggregation have not yet been fully identified. For instance, the reduction in the rate of $A \beta 42$ aggregation observed in the presence of dipalmitoylphosphatidylcholine (DPPC) gel phase membranes ${ }^{25}$, was abolished upon addition of cholesterol to form liquid ordered bilayer membranes ${ }^{25}$. On the other hand, other studies suggested that cholesterol can inhibit interactions between $A \beta$ and lipid membranes, thus increasing the extracellular levels of $A \beta$ and promoting its aggregation ${ }^{26}$. 
In order to investigate this problem, here we analyse quantitatively the direct effects of cholesterol on AB42 aggregation using the strategy illustrated in Fig. 1. Our approach exploits the recent development of highly reproducible thioflavin-T (ThT)based kinetic measurements and of analytical approaches to their intepretations ${ }^{27,28}$. Such measurements have made it possible to show that the aggregation of $A \beta 42$ is characterised by a surface-catalysed secondary nucleation mechanism, whereby the fibril surfaces act as catalytic sites for the generation of toxic $A \beta 42$ oligomers. The oligomers thus generated can grow further and convert into fibrils, promoting the formation of additional toxic species in a highly effective catalytic cycle $e^{28-34}$.

The analysis of the reaction kinetics has been powerful also in characterising the effects of intrinsic and extrinsic factors on the rates of individual microscopic steps in the aggregation process of the $A \beta 42$ peptide ${ }^{35-39}$. In particular, the diverse pathways through which macromolecules, such as molecular chaperones and antibodies, can affect the aggregation of the $A \beta 42$ peptide have recently been described in detail ${ }^{35-}$ 37,40. Moreover, using the same approach, the effects of disease-associated mutations and $\mathrm{pH}$ variations could be deciphered, showing that both factors can give rise to enhancements in the secondary nucleation process of $A \beta 42$ aggregation ${ }^{32}$. It has also been shown that the effects of small molecules on the different microscopic steps of the $A \beta 42$ aggregation process can also be monitored in detail, thus leading to the development of a chemical kinetics-based drug discovery strategy that aims at identifying drug candidates that can inhibit specific steps in $A \beta 42$ aggregation ${ }^{41,42}$.

In the present study, we extend this kinetics-based strategy to decipher the role of cholesterol in modulating $A \beta 42$ aggregation. To this end, we characterise at the molecular level the effects of vesicles prepared with 1,2-dimyristoyl-sn-glycero-3phosphocholine and cholesterol (DMPC:cholesterol vesicles) on the microscopic steps of $A \beta 42$ aggregation (Fig. 1). Our results indicate that these vesicles provide a model membrane that can effectively increase the nucleation rate of $A \beta 42$. Furthermore, we show that despite the occurrence of a cholesterol-dependent nucleation process, surface-catalysed secondary nucleation remains the dominant mechanism for aggregate proliferation, as in the lipid-free aggregation process. We then provide evidence that primary oligomers of $A \beta 42$, whether they are formed 
through homogeneous or heterogeneous nucleation events, are likely to possess structural similarities by showing that a previously characterised inhibitor of the homogeneous nucleation of A $\beta 42$ aggregation also inhibits the heterogeneous primary nucleation events catalysed by lipid vesicles ${ }^{41}$.

Overall, these results reveal a self-assembly process that includes a heterogeneous primary nucleation step through which $A \beta 42$ aggregates in the presence of DMPC:cholesterol vesicles. This step can be significantly faster than that of the homogeneous primary nucleation, leading to the more rapid formation of potentially neurotoxic $A \beta 42$ oligomers. We have therefore proposed a mechanism to explain how the presence of cholesterol can accelerate $A \beta 42$ aggregation, thus enhancing our understanding of the molecular origins of $A D$ and helping the development of effective therapeutics against this devastating disease.

\section{Results}

Cholesterol-containing lipid vesicles accelerate $A \beta 42$ aggregation. To decipher the role of cholesterol in $A \beta 42$ aggregation, we carried out a global kinetic analysis of $A \beta 42$ aggregation $^{27}$ in the presence of zwitterionic vesicles containing cholesterol at concentrations ranging from 0 to $40 \mathrm{~mol} \%$ of the total lipid concentration (Fig. 2 and Supplementary Figs. 1-4). We investigated this range because cholesterol accounts form about $20 \%$ of the total content of brain lipids, although its levels in neuronal membranes can vary substantially from one cell or organelle to another ${ }^{43,44}$. Thus, for example, the outer monolayer of plasma membranes have cholesterol levels of around $30 \mathrm{~mol} \%$, while they are much lower in the corresponding inner monolayer ${ }^{45}$.

Since phosphatidylcholine (PC) lipids, together with phosphatidylethanolamine (PE) and phosphatidylserine (PS) lipids, constitute the most abundant components of neuronal membranes ${ }^{46}$, we investigated the effects of cholesterol on $A \beta 42$ aggregation for a series of model systems composed of zwitterionic PC lipids with different acyl-chain compositions in terms of the length and degree of unsaturation (DMPC (2 chains C14:0), POPC (C18:1 \& C16:0) and DOPC (2 chains C18:1)). We 
found that the level of unsaturation alters to different extents the effects of cholesterol on $A \beta 42$ aggregation, with the rate of aggregation increasing with the degree of unsaturation of the PC hydrocarbon chain (DOPC>POPC>DMPC) (Supplementary Figs. 1 and 4). These findings may be explained by the increased exposure to hydrophobic hydrocarbon layer of the bilayer for the unsaturated lipids with bulky hydrocarbon chains, as the average accessible area per $P C$ headgroup in the bilayer varies from $60 \AA^{2}$ for DMPC to $69 \AA^{2}$ for POPC and $72 \AA^{2}$ for DOPC ${ }^{47,48}$. By contrast, although anionic lipids, such as PS, can influence significantly $A \beta 42$ aggregation and the structure of the aggregates ${ }^{49,50}$, they are mainly found in the inner leaflet of the plasma membrane, and therefore are not enriched in the regions where cholesterol is primarily located.

Therefore, to assess specifically the effects of cholesterol on the aggregation of $A \beta 42$ systematically in a physiologically relevant system, we used model systems composed of DMPC and cholesterol, which capture several important features of the behaviour of cholesterol in membranes highly relevant to lipid rafts ${ }^{51}$, including the formation of small scale domains and of the liquid ordered membrane structure ${ }^{52-54}$. The advantage of these model systems is that DMPC alone does not significantly influence $A \beta 42$ aggregation (Supplementary Fig. 1), making it possible to identify clearly the effects of the added cholesterol.

Using DMPC:cholesterol vesicles, we found that increasing cholesterol content significantly enhanced $A \beta 42$ aggregation (Fig. 2 and Supplementary Figs. 1-3). These results also show that the effect of the lipid vesicles on the aggregation behaviour depends on both the lipid-to-protein (L/P) ratio (mol:mol) (Fig. 2b) and the cholesterol content within the membrane (Fig. 2c). We also observed a similar behaviour when we added cholesterol to model membranes composed of DMPC and DMPE, further supporting the conclusion that the presence of cholesterol promotes

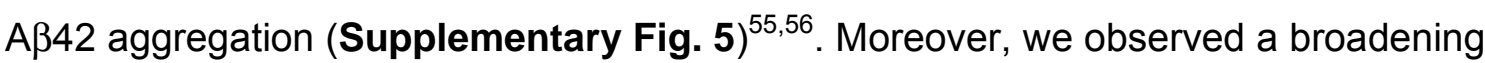
of the DMPC melting transition and an increase in the size and fluidity of the DMPC vesicles with increasing concentrations of cholesterol (Supplementary Figs. 6 and $7)^{57,58}$. In addition, the acceleration of the aggregation kinetics of $A \beta 42$ was not directly related to an increase in the size of the vesicles (Supplementary Fig. 8). 
A maximum effect corresponding to a decrease by about $35-40 \%$ of the half time of the aggregation reaction was observed at $[\mathrm{L}] /[\mathrm{P}]$ ratios above 200 for vesicles containing $5 \%$ and $10 \%$ cholesterol (Fig. $\mathbf{2 b}$ ). For all $[\mathrm{L}] /[\mathrm{P}]$ ratios, the acceleration in the aggregation kinetics of $A \beta 42$ shows a strong dependence on the membrane composition up to a cholesterol content of about $15 \mathrm{~mol} \%$; the acceleration appears to be unaffected by further increases (Fig. 2 and Supplementary Figs. 1-3). This non-linear response of the aggregation kinetics suggests that specific changes in the membrane properties are crucial to the aggregation process ${ }^{59,60}$. These results can be rationalised by observing that in a PC lipid system at $37{ }^{\circ} \mathrm{C}$, a single liquid disordered $\left(I_{d}\right)$ phase is present at cholesterol contents below $15 \%$. The cholesterol molecules are homogenously solubilized in the bilayer, gradually changing its properties and composition with increasing cholesterol contents (Fig. 2c). When the cholesterol content exceeded $15 \mathrm{~mol} \%$, the system segregated into small-scale domains ("rafts") composed of $I_{d}$ and liquid ordered $\left(I_{0}\right)$ phases. At a cholesterol content above $30 \mathrm{~mol} \%$, however, a single $I_{0}$ was formed, and over the whole range of domain formation, the compositions in the segregated $I_{d}$ and $I_{o}$ domains were unchanged and determined by the compositions at the phase boundaries, with the proportions of the different domains varying with cholesterol content.

To exclude possible interferences of the lipid vesicles with the ThT fluorescence signal, $A \beta 42$ aggregation was also monitored by circular dichroism (CD) spectroscopy (Supplementary Fig. 9) ${ }^{61}$. We recorded time-dependent CD spectra of a $20 \mu \mathrm{M}$ A $\beta 42$ solution in the absence or presence of DMPC vesicles with either $0 \%$ or $15 \%$ cholesterol at a $[\mathrm{L}] /[\mathrm{P}]$ ratio of 10 (Supplementary Fig. 9). At time 0 , the $C D$ spectrum of $A \beta 42$ was characterised by a negative mean residue ellipticity (MRE) at about $200 \mathrm{~nm}$, which is characteristic of an unfolded peptide. At the end of the reaction, however, the spectrum exhibited positive and negative MRE values at about $200 \mathrm{~nm}$ and $218 \mathrm{~nm}$, characteristic of $\beta$-sheet structure. The time dependence of the MRE values at $218 \mathrm{~nm}\left(\mathrm{MRE}_{218 \mathrm{~nm}}\right)$ in the presence of vesicles at a $[\mathrm{L}][\mathrm{P}]$ ratio of 10 shows that $\beta$-sheet formation occurs at a slightly slower rate in the presence of DMPC vesicles than in its absence (Supplementary Fig. 9). This difference could arise from the multiple general contributions of foreign surfaces provided by both the cuvette and the lipid, which can affect the aggregation process ${ }^{62}$. By comparing the 
spectra over time in the presence of DMPC:cholesterol vesicles containing $15 \%$ cholesterol, and in the presence of vesicles free of cholesterol, where general additional contributions from surface effects are similar, we observed that the formation of $\beta$-sheet structures of $A \beta 42$ occurs more rapidly in the presence of DMPC:cholesterol vesicles, in agreement with the results obtained using ThT-based kinetics (Supplementary Fig. 9).

\section{Morphology and structure of $A \beta 42$ fibrils in the presence of cholesterol-} containing lipid vesicles. Using atomic force microscopy (AFM) we acquired highresolution 3D morphology maps of $A \beta 42$ fibrils formed in the absence and in the presence of DMPC vesicles, the latter both free of cholesterol or containing $15 \%$ cholesterol at a $[\mathrm{L}] /[\mathrm{P}]$ ratio of 10 (Fig. 3a) ${ }^{63}$. No significant differences in the length and height of the fibrils could be observed between the samples. Also, by using cryoelectron microscopy (cryo-EM), we found that fibrils formed in the presence of vesicles with $15 \%$ cholesterol (Fig. $\mathbf{3 b}, \mathbf{c}$ ) are of similar length and thickness as those formed of A $\mathrm{\beta} 42$ alone (Fig. 3d,e). However, the fibrils appeared to be organised slightly differently, implying that they could have altered surface properties. Previous observations of the presence of lipids in amyloid plaques ${ }^{10}$ suggest that lipids may be incorporated in the aggregates; indeed few vesicles are seen (Fig. 3b,c). To investigate further the structural organisation of the fibrillar aggregates, we used Fourier transform infrared (FTIR) spectroscopy (Fig. 3f and Supplementary Fig. 10). The IR absorption of proteins is characterised by a series of amide bands, with the shape and position of amide bands I providing information on their secondary and quaternary conformations ${ }^{64}$. We found identical amide I bands, and thus similar structural organization of the ThT-free A $\beta 42$ fibrils within experimental errors, as also demonstrated by the comparison of their second derivatives (Fig. 3f and Supplementary Fig. 10). In addition, we carried out fluorescence measurements that showed that the spectra of ThT bound to A $\beta 42$ fibrils are similar in the presence and absence of the cholesterol-containing lipid vesicles during fibril formation (Supplementary Fig. 10). 


\section{DMPC:cholesterol vesicles accelerate the rate of $A \beta 42$ primary nucleation by}

up to 20-fold. We carried out a quantitative analysis of $A \beta 42$ aggregation in the presence of lipid vesicles to define the changes in the rate constants governing each microscopic step. The analysis of $t_{\text {lag }}$ (the time required for ThT fluorescence to reach $10 \%$ of the final value) and $t_{\text {growth }}$ (the time required for ThT fluorescence to reach $90 \%$ of the final value after $t_{\text {lag }}$ ) of $A \beta 42$ aggregation shows that these vesicles have a concentration-dependent effect on $t_{\text {lag }}$ but no significant effect on $t_{\text {growth }}$ (Fig. 4a). These results indicate that the primary nucleation pathway is the main microscopic process perturbed by the presence of DMPC:cholesterol vesicles ${ }^{65,35}$. In addition, the scaling exponent of the $A \beta 42$ concentration dependence, which reflects the dominance of the fibril-catalysed secondary nucleation process ${ }^{29}$, remained unaffected within error limits with a value close to -1.3 in the presence of these vesicles (Fig. 4b).

We then carried out an additional series of measurements of the aggregation kinetics of A 342 under conditions where the surface-catalysed secondary nucleation was supressed by a molecular chaperone, the Brichos domain ${ }^{37}$ (Fig. 4c and Supplementary Fig. 11). Thus, in the presence of Brichos, $A \beta 42$ aggregation is mainly driven by primary nucleation and elongation since surface-catalysed secondary nucleation is completely suppressed (Supplementary Fig. 11). We found that the aggregation kinetics of $A \beta 42$ were significantly accelerated in the presence of DMPC:cholesterol vesicles, and to a much smaller extent when the vesicles are free of cholesterol (Fig. 4c). Thus, the observed effects can be attributed to an increase in the rates of primary nucleation and/or elongation when cholesterol is incorporated in the vesicles.

Next, to define more quantitatively how cholesterol affects the microscopic rates, we modified the underlying kinetic equations (Eqs. 1 and 2) to account for the addition of a lipid-induced aggregation process to the three microscopic events (primary nucleation, surface-catalysed secondary nucleation and elongation) involved in the aggregation pathway of $A \beta 42$. We analysed the aggregation profiles using these modified equations, which relate the macroscopic time evolution of the fibril mass to the rate constants of the different microscopic events. We found the best fit when the 
rate of the primary pathway $\left(k_{+} k_{n}\right)$ is significantly increased, while the secondary pathway $\left(k_{+} k_{2}\right)$ was relatively unaffected (Fig. $\left.4 \mathbf{d}-\mathbf{e}\right)$. These results indicate that DMPC:cholesterol vesicles are mainly involved in enhancing the primary nucleation process, rather than the elongation process. Similar results were obtained for DMPC:cholesterol vesicles containing cholesterol levels as low as $5 \%$ (Supplementary Fig. 12). Additionally, the best fit of the $A \beta 42$ aggregation kinetics in the presence of Brichos and DMPC:cholesterol vesicles corresponds to an increase only in the rate of the primary nucleation, thus further confirming the role of DMPC:cholesterol vesicles in accelerating the primary processes in A $\beta 42$ aggregation (Supplementary Fig. 11).

Taken together, these data indicate that the DMPC:cholesterol vesicles act as catalysts of $A \beta 42$ aggregation by increasing the formation of primary oligomers thereby accelerating the overall aggregation kinetics. This cholesterol introduces heterogeneous primary nucleation as a new active process into the $A \beta 42$ aggregation pathway. Thus, the primary nucleation of $A \beta 42$ occurs through two distinct mechanisms, a homogenous pathway (with rate constant $k_{n}$ ) where new aggregates are formed solely from the interactions between soluble monomers, and a heterogeneous pathway where new aggregates are generated at a rate that depends on the concentrations of both monomeric $A \beta 42$ and lipid vesicles (with rate constant $\left.k_{\text {cat }}\right)$. Hence, the overall rate constant of $\mathrm{A} \beta 42$ primary nucleation becomes $k_{\mathrm{n}}{ }^{\prime}$, which accounts for the contribution of both $k_{\mathrm{n}}$ and $k_{\text {cat }}$ (see Materials and Methods).

Heterogeneous primary nucleation depends on the concentration of $\mathrm{A} \beta 42$ monomers and on the ratio of accessible sites, $s$, of the DMPC:cholesterol vesicles. Indeed, a steep linear increase with the $[\mathrm{L}][\mathrm{P}]$ ratio in the primary pathways was observed for DMPC:cholesterol vesicles containing $10 \%$ cholesterol at a [L]/[P] ratios below 200 (Fig. 4e). In the case of DMPC:cholesterol vesicles containing $5 \%$ cholesterol, saturation could not be observed even in the presence of $[\mathrm{L}] /[\mathrm{P}]$ ratios as high as 400 (Supplementary Fig. 12). In all cases, the heterogeneous primary nucleation was found to be linearly dependent on the $[\mathrm{L}][\mathrm{P}]$ ratio, and consequently on the ratio of accessible sites, $s$, before saturation is reached (see Materials and Methods). At 
high concentrations of accessible sites, i.e. at $[\mathrm{L}][\mathrm{P}]$ ratios above 200 in the case of DMPC:cholesterol vesicles containing 10\% cholesterol, where the heterogeneous primary nucleation is likely to be restricted by the adsorption and desorption rate of the peptide, the relative increase in the heterogeneous primary nucleation rate is maximal and corresponds to an increase by a factor of $20 \pm 2$ (Fig. 4e). We next carried out a numerical estimate of the rate of the formation of the total mass of $A \beta 42$ aggregates in which we increased the rate of primary nucleation by 20 -fold (Fig. 4f). In agreement with the results of all the experiments performed in the presence of sufficient amounts of cholesterol to achieve maximum rate of $A \beta 42$ aggregation (Fig. 4f, inset), we found that the theoretical half-time is smaller than that of $A \beta 42$ alone by about $40 \%$ (Figs. $\mathbf{2 b}$ and $\mathbf{4 f}$ ). A theoretical prediction based on the analytical solution for the aggregation kinetics ${ }^{6-68}$ indicates that an increase in the rate of primary nucleation of an aggregation reaction translates into a decrease of about $1 / \log (20)$, i.e. $34 \%$, in the reaction half-time, which is fully consistent with the experiments reported here (see Materials and Methods).

\section{Chemical kinetics characterise the roles of A 342 monomers and cholesterol in} DMPC:cholesterol vesicles in the heterogeneous primary nucleation step. The relative acceleration, $\varepsilon$, of primary nucleation in $A \beta 42$ aggregation due to the introduction of a lipid-associated heterogeneous pathway is dependent on the concentration of A $\beta 42$ monomers, $m(0)$, as well as the accessibility factor, $\sigma$, of the lipid system, which we found to be dependent on the proportion of cholesterol within the lipid membrane (Eq. 9). We thus set out to characterise in detail the contributions to the heterogeneous primary nucleation pathway of the various species involved, including the AB42 monomers and the fraction of cholesterol in DMPC:cholesterol vesicles.

First, we observed that the total lipid concentration of the DMPC:cholesterol vesicles containing $10 \%$ cholesterol required for a maximum rate of $A \beta 42$ aggregation was higher at increasing concentrations of $A \beta 42$ (Supplementary Fig. 13). This observation implies that $\varepsilon$ decreases as the concentration of $A \beta 42$ monomers decreases. Considering that $\alpha$ (Eq. 10) describes the relative increase of primary nucleation due to the additional heterogeneous primary nucleation per lipid surface 
available for each peptide, a double logarithmic plot of $\alpha m(0)^{n_{c}}$ against the initial monomer concentration, $m(0)$, yields a line with slope $n_{\mathrm{h}}$, which corresponds to the monomer concentration dependency of the heterogeneous nucleation pathway (Fig. 5a, Materials and Methods). The low reaction order observed for $n_{\mathrm{h}}\left(n_{\mathrm{h}}<2\right)$ suggests that, over the range of monomer concentrations probed in our experiments (1.5-3 $\mu \mathrm{M})$, the coverage of a single nucleation site by monomers could be saturated, such that the heterogeneous nucleation pathway in this case is primarily dependent on the number of accessible sites for each peptide ([L]/[P]) (see Materials and Methods).

As observed above, the maximum rate of $A \beta 42$ aggregation occurred at different $[L] /[P]$ ratios for the different cholesterol content of DMPC:cholesterol vesicles (Fig. 2b,c and Supplementary Figs. 1-3). These effects were found to depend in a nonlinear manner on the cholesterol concentration, thus suggesting that the organisation of cholesterol within the DMPC:cholesterol membranes might be responsible for creating accessible catalytic sites, $s$, for $A \beta 42$ nucleation. To explain these findings, we hypothesised that the relative increase of primary nucleation, $\alpha$, might be controlled by the presence of multiple cholesterol molecules. From the probability of observing $m$ adjacent sites occupied by cholesterol molecules, $p=\theta^{m}$, where $\theta$ denotes the surface coverage by cholesterol, i.e. the percentage of cholesterol within the membrane, we found for the accessibility factor, $\sigma \sim \theta^{m}$. To test this prediction, we plotted $\alpha$ as a function of $\theta$ in a log-log plot, finding a line with a slope of $m>1$ (Fig. 5b). This result indicates that the number of accessible catalytic surface sites is correlated with the higher order assembly of cholesterol molecules within the DMPC:cholesterol membrane. Therefore, under these conditions, at the maximal acceleration of A $\beta 42$ aggregation, where the primary pathways are accelerated 20fold, assuming that the catalysis involves several molecules of cholesterol, the total number of catalytic sites is approximately $10 \%$ of the number of $A \beta 42$ monomers (see Materials \& Methods). These results imply the presence of a positive cooperativity in the interaction of $A \beta 42$ with cholesterol-containing membranes, and further increases in the number of catalytic sites do not increase the aggregation rate. The total number of nucleation sites estimated in this way was found to be at 
least two orders of magnitude higher than the number of nucleation sites required for a maximal effect to occur.

We then explored whether or not $A \beta 42$ oligomers formed as a result of heterogeneous primary nucleation possess similar structural features to those formed by homogeneous primary nucleation. Since primary nuclei are formed transiently during the aggregation process, their direct structural characterisation is very challenging ${ }^{6}$. We therefore assessed their structural properties using as a probe bexarotene, a small molecule that inhibits $A \beta 42$ primary nucleation (Fig. 5c and Supplementary Fig. 14) $)^{41}$. We found that bexarotene inhibits $A \beta 42$ primary nucleation irrespective of whether the primary oligomers of $A \beta 42$ are formed through homogeneous or heterogeneous nucleation. These results suggest that the nuclei generated through homogeneous or heterogeneous primary nucleation share common structural features (Fig. 5c and Supplementary Fig. 14).

We then estimated the total rate of formation of oligomers from both primary and secondary processes in the aggregation of a $2 \mu \mathrm{M}$ solution of $A \beta 42$ in the absence and presence of DMPC:cholesterol vesicles containing increasing concentrations of cholesterol (see Materials and Methods). We found that increasing the rate of primary nucleation increases the aggregation reaction rate without affecting significantly the total load of toxic oligomers generated during the reaction ${ }^{29,35-37,41,42}$. Consistently, our calculations show that accelerating $A \beta 42$ primary nucleation in the presence of DMPC:cholesterol vesicles is accompanied by earlier formation of oligomers (Fig. 5d).

\section{Discussion}

It is increasingly recognised that changes in lipid homeostasis can influence the levels of toxic species formed by $A \beta 42$, as indicated by recent results both in cell cultures and in vivo ${ }^{22,69}$. Moreover, the kinetics of $A \beta 42$ oligomerization and amyloid formation in vitro have been found to be affected by the presence of specific lipids including gangliosides and sphingomyelin ${ }^{70}$. 
To rationalise these findings, we have identified here a mechanism by which a specific lipid involved in $A D$ pathology influences the process of $A \beta 42$ aggregation. We have thus shown that cholesterol-containing vesicles provide an alternative heterogeneous primary nucleation route that results in a faster rate of formation of oligomeric species.

These results provide insights into the potential pathological role of $A \beta 42$ in brain tissue. For typical cellular volumes (0.3-5 pL) and physiological levels of $A \beta 42$ (1-10 $\mathrm{nM}$ ), based on Eq. 6, we can estimate that it would take on average between 10 and 300 years for the $A \beta 42$ aggregation process to occur spontaneously. We found, however, that the onset of $A \beta 42$ aggregation is accelerated up to 20 times by the heterogeneous primary nucleation pathway created by DMPC:cholesterol vesicles, thus dramatically reducing the average nucleation times to the range of months to decades. Although these estimates are only indicative, as they do not reflect the complexity of the cellular environment, they illustrate how heterogeneous nucleation processes can substantially accelerate the formation of A 42 species (Fig. 6).

Overall, our results indicate that while spontaneous $A \beta 42$ aggregation may be a rather slow process, triggering it through heterogeneous nucleation pathways can increase substantially the rate of aggregation, suggesting that cholesterol, or other compounds with a similar effect on $A \beta 42$ aggregation, could indirectly constitute a risk factor for $A D$.

By describing a molecular mechanism through which DMPC:cholesterol vesicles promote $A \beta 42$ aggregation, we have provided a possible explanation for the proposed link between increased levels of cholesterol and AD. These results have been obtained by applying a chemical kinetics approach that allowed us to dissect the overall macroscopic aggregation process of $A \beta 42$ into its underlying component microscopic reactions. This strategy has led to the determination of the manner in which DMPC:cholesterol vesicles contribute to the early steps in A $\beta 42$ aggregation by providing an alternative route for primary nucleation. The present work thus provides a clear example of the importance of understanding in detail the molecular 
mechanisms in which physiological factors can trigger the aggregation of $A \beta 42$ in the complex molecular environment of the brain. As we have shown these factors can dramatically reduce the typical timescale of $A \beta 42$ aggregation, and thus their identification is crucial to understand the best points of intervention for strategies aimed at blocking this process.

\section{Methods}

\section{Theoretical analysis}

Kinetic analysis in the absence of lipids. The aggregation of $A \beta 42$ involves a process where secondary nucleation is responsible for the generation of most of the aggregates $^{29}$. The aggregate mass concentration, $M(t)$, and the aggregate number concentration, $P(t)$, can be described by the following differential equations ${ }^{31}$ :

$$
\begin{aligned}
& \frac{d P(t)}{d t}=k_{n} m(t)^{n_{c}}+k_{2} m(t)^{n_{2}} M(t) \\
& \frac{d M(t)}{d t}=2 m(t) k_{+} P(t)
\end{aligned}
$$

where the initial concentration of soluble monomers is denoted by $m(0), n_{\mathrm{c}}$ and $n_{2}$ describe the dependencies of the primary and secondary pathways on the monomer concentration, and $k_{n}, k_{+}$and $k_{2}$ are the rate constants of primary nucleation, elongation and surface-catalysed secondary nucleation, respectively.

Using self-consistent methods, approximate analytical solutions to these differential equations can be obtained that describe the time evolution of the total fibril mass concentration, $M(t)$, as ${ }^{29,30}$

$$
\frac{M(t)}{M(\infty)}=1-\left(\frac{B_{+}+C_{+}}{B_{+}+C_{+} e^{\kappa t}} \frac{B_{-}+C_{+} e^{\kappa t}}{B_{-}+C_{+}}\right)^{\frac{k_{\infty}^{2}}{\kappa \overline{\phi_{\infty}}}} e^{-k_{\infty} t}
$$


where $M(\infty)=m(0)$ is the final mass concentration of aggregates and

$$
B_{ \pm}=\frac{k_{\infty} \pm \widetilde{k_{\infty}}}{2 \kappa}, \quad C_{ \pm}= \pm \frac{\lambda^{2}}{2 \kappa^{2}}, \quad k_{\infty}=\sqrt{\frac{2 \kappa^{2}}{n_{2}\left(n_{2}+1\right)}+\frac{2 \lambda^{2}}{n_{c}}}, \widetilde{k_{\infty}}=\sqrt{k_{\infty}{ }^{2}-4 C_{+} C_{-} \kappa^{2}}
$$

with $\lambda=\sqrt{2 k_{+} k_{n} m(0)^{n_{c}}}$ and $\kappa=\sqrt{2 k_{+} k_{2} m(0)^{n_{2}+1}}$.

Note that, according to Eq. 3, to capture the complete macroscopic assembly process, only two particular combinations of the rate constants are needed, namely $k_{+} k_{n}$ and $k_{+} k_{2}$. These two particular combinations of microscopic rate constants define the intrinsic timescales associated with the proliferation of new aggregates through primary pathways, $\lambda=\sqrt{2 k_{+} k_{n} m(0)^{n_{c}}}$, and through secondary pathways, $\kappa=$ $\sqrt{2 k_{+} k_{2} m(0)^{n_{2}+1}}$, respectively.

The experimental curves that are shown in Fig. 4c-d and Supplementary Fig. 12 were fitted using Eq. 3 where we introduced perturbations to $\lambda$ and $\kappa$ in order to account for the inclusion of the lipid-surface catalysed process. This procedure is carried out by adding to the rate constants of each of the microscopic steps a new factor, $\mathrm{x}$, that allows the description of the experimental curves in the presence of the lipids, i.e. $k^{\prime}=k+\mathrm{x}$. It was found that the best fits were derived when the rate of primary pathways $\left(k_{+} k_{n}\right)$ was significantly increased, and the rate of secondary pathways $\left(k_{+} k_{2}\right)$ was relatively unaffected. Coupled with other experiments (Fig. 4ac) that support these fits, we observe that the lipid-catalysed process is mainly captured in the primary pathway, $\lambda$.

Theoretical model for a lipid-catalysed heterogeneous primary nucleation. The presence of low amounts of DMPC:cholesterol vesicles introduces a new active process into the aggregation pathway of $A \beta 42$, known as heterogeneous primary nucleation with the $k_{\text {cat }}$ rate constant:

$$
\begin{aligned}
& \frac{d P(t)}{d t}=k_{n} m(t)^{n_{c}}+k_{c a t} m(t)^{n_{h}} s+k_{2} m(t)^{n_{2}} M(t) \\
& s=\sigma\{[L] /[P]\}
\end{aligned}
$$


where $s$ denotes the ratio of accessible surface sites for heterogeneous primary nucleation, which is a function of the lipid-to-protein ratio, $[\mathrm{L}] /[\mathrm{P}] . \sigma$ is the surface accessibility constant, and $n_{\mathrm{h}}$ denotes the monomer concentration dependency of the heterogeneous nucleation pathway. It should be noted that the surface accessibility factor $\sigma$ is dependent on the lipid system. In this particular study, the parameter $\sigma$ is found to depend on the fraction of cholesterol in the DMPC:cholesterol membrane (Fig. 2b-c); in particular, $\sigma$ is much greater in the case of DMPC:cholesterol vesicles as compared to the cholesterol-free ones. We rationalise the heterogeneous nucleation mechanism through the assumptions of the Langmuir adsorption isotherm, which indicates that the available lipid vesicles provide a surface for adsorption of A $\beta 42$. This may facilitate nucleation and lead to the formation of $A \beta 42$ primary oligomers on the surface of the DMPC:cholesterol vesicles.

Generalising the self-consistent methods to the new kinetic equations, it is possible to obtain an approximate analytical solution for $M(t)$ in the presence of the additional nucleation mechanism (Eq. 4) as

$$
\frac{M(t)}{M(\infty)}=1-\left(\frac{B_{+}+C \prime_{+}}{B \prime_{+}+C \prime_{+} e^{\kappa \prime t}} \frac{B_{-}+C_{\prime_{+}} e^{\kappa \prime t}}{B \prime_{-}+C_{+}}\right)^{\frac{k \prime_{\infty}^{2}}{\kappa \prime \overline{k \prime} \infty}} e^{-k_{\infty}^{\prime} t}
$$

where $M(\infty)=m(0)$ as before and

$$
B_{ \pm}^{\prime}=\frac{k_{\infty}^{\prime} \pm \widetilde{k_{\infty} \infty}}{2 \kappa^{\prime}}, \quad C_{ \pm}^{\prime}= \pm \frac{\lambda \prime^{2}}{2 \kappa^{\prime}{ }^{2}}, \quad k_{\infty}^{\prime}=\sqrt{\frac{2 \kappa^{\prime 2}}{n_{2}\left(n_{2}+1\right)}+\frac{2 \lambda^{\prime 2}}{n_{c}}}, \quad \widetilde{k_{\infty}^{\prime}}=\sqrt{k_{\infty}^{\prime}{ }^{2}-4 C^{\prime}{ }_{+} C^{\prime}{ }_{-} \kappa^{\prime 2}}
$$

with $\kappa^{\prime}=\kappa=\sqrt{2 k_{+} k_{2} m(0)^{n_{2}+1}}$ and with a slight modification to $\lambda$, the primary pathway

$\lambda^{\prime}=\sqrt{2 k_{+} k_{n} m(0)^{n_{c}}+2 k_{+} k_{c a t} m(0)^{n_{h}}}$

Hence, the additional heterogeneous nucleation mechanism can be accounted for by a modification of the rate constant of primary nucleation, $k_{n}$, to give an effective rate constant, $k_{\mathrm{n}}$, that includes the heterogeneous nucleation pathway 


$$
k_{n}^{\prime}=k_{n}+k_{c a t} m(0)^{n_{h}-n_{c} S}
$$

In other words, the analytical solution in Eq. 6 is equivalent to Eq. 3 with a slight modification to $\lambda$ that accounts for the presence of the additional nucleation pathway. Hence, kinetic traces of aggregation in the presence of heterogeneous primary nucleation can be analysed simply by using Eq. 3 (the analytical solution in the absence of lipids), but the extracted combined parameter for the primary pathway, $k_{+} k_{n}{ }^{\prime}$, is interpreted, using Eq. 8, as an effective parameter that includes the effect from the heterogeneous nucleation step.

Assuming that the homogeneous primary nucleation rate is unaffected by the introduction of the heterogeneous nucleation pathway, using Eq. 8, we can compute the (relative) acceleration $\varepsilon=\frac{k_{n}^{\prime}}{k_{n}}$ of primary nucleation due to the presence of a heterogeneous pathway as

$\varepsilon=\frac{k_{n}^{\prime}}{k_{n}}=1+\frac{\left.\sigma k_{c a t} m(0)\right)^{\left(n_{h}-n_{c}\right)}[L] /[P]}{k_{n}}$

and it is therefore dependent on the concentration of $A \beta 42$. For a given lipid system where the accessibility factor, $\sigma$, is constant for various protein concentrations, the slope $\alpha$ of a plot of $\frac{k_{n}^{\prime}}{k_{n}}$ vs the lipid-to-protein ratio $[\mathrm{L}] /[\mathrm{P}]$

$$
\alpha=\frac{\sigma k_{c a t} m(0)\left(n_{h}-n_{c}\right)}{k_{n}}
$$

can be used to determine the reaction order for the heterogeneous nucleation step, $n_{\mathrm{h}}$. Specifically, the slope of a double logarithmic plot of $\operatorname{com}(0)^{n_{c}}$ against $m(0)$ equals $n_{\mathrm{h}}$ (Fig. 4a). The low reaction order observed for $n_{\mathrm{h}}$ suggests that over the range of monomer concentrations probed in our experiments $(1.5-3 \mu \mathrm{M})$, the coverage of monomers along a single nucleation site could be saturated. Specifically, we can model the rate of heterogeneous primary nucleation using a Langmuir adsorption isotherm model by replacing $m(0)$ by the monomer surface coverage 
$m(0)_{c o v}=\frac{m(0)}{K+m(0)}$, where $\mathrm{K}$ is the equilibrium binding constant. When the coverage of the nucleation sites by the monomers is large $(m(0)>>\mathrm{K})$, the Langmuir adsorption process is saturated, meaning that $m(0)_{c o v}$ is independent of $m(0)$; hence, the saturation of surface nucleation sites could explain the observed low reaction order $n_{\mathrm{h}}$ of the heterogeneous nucleation pathway with respect to the monomer concentration.

In the presence of large number of accessible surface sites, the rate of heterogeneous primary nucleation is constrained by the availability of free $A \beta 42$ monomers that could adsorb to a surface nucleation site at any point of time, rather than by the lipid concentration, so the rate laws become:

$$
k_{n}{ }^{\prime}=k_{n}+k_{\text {cat }} \max
$$

where $k_{\text {cat }} \max =k_{c a t} s_{\max }$, and

$$
\frac{k_{+} k_{n^{\prime}}}{k_{+} k_{n}}=1+\frac{k_{c a t} \max }{k_{n}}
$$

By taking the maximum increase of $k_{+} k_{n}{ }^{\prime} / k_{+} k_{n}$ into account (Fig. 4f), we can conclude that the contribution of the heterogeneous primary nucleation increases the overall rate of primary nucleation by maximum 20 -fold (Eq. 7 ).

From the approximate analytical solution Eq. 3, $t_{1 / 2}$ is found to occur at ${ }^{66-68}$

$$
t_{1 / 2} \approx \log \left(\frac{1}{C_{+}}\right) \kappa^{-1}
$$

Thus, if we consider solely an increase in the rate of primary nucleation, $k_{\mathrm{n}}$ ' by 20 times, $\lambda$, the primary nucleation pathway, would increase by approximately $\sqrt{20}$ times. Correspondingly, $C_{+}=\frac{\lambda^{2}}{2 \kappa^{2}}$ would increase by approximately 20 times, with no change in $\kappa$. Hence, the relative increase in $t_{1 / 2}$ is estimated to be $\log (1 / 20) \approx 34 \%$. 
Estimate of the number of nucleation sites. Under the assumption of random and independent packing of cholesterol molecules on the surface of lipid vesicles, the probability of finding $m$-tuples of occupied adjacent surface sites is:

$p=\theta^{m}$

where $\theta$ is the surface coverage by cholesterol, i.e. the fraction of surface sites covered by cholesterol molecules, that is the $\%$ of cholesterol within the membrane. Since $s=\sigma\{[L] /[P]\}$ (Eq. 5), $\sigma \sim \theta^{m}$.

As we defined earlier (Eq. 10), the slope $\alpha$ of a plot of $\frac{k_{n}^{\prime}}{k_{n}}$ vs lipid concentration [L] depends on both $\sigma$ and the concentration of a monomer. Thus, for a constant protein concentration of different lipid systems (DMPC with different proportions of cholesterol), $\alpha$ can be used to determine $\sigma$. Since $\sigma \sim \theta^{m}$, the slope of a double logarithmic plot of $\alpha$ against the $\%$ of cholesterol within the membrane, $\theta$, we find that $m \approx 3$ according to Fig. $\mathbf{5 b}$.

At given monomer and lipid concentrations, we can thus estimate the number of accessible surface nucleation sites per A $\beta 42$ monomer

$r_{\text {sites }}=\{P(s)[$ lipid $]\} / m(0)$

By taking the $[\mathrm{L}] /[\mathrm{P}]$ ratio at which maximal acceleration of $2 \mu \mathrm{M} A \beta 42$ aggregation is observed for the different cholesterol concentrations, the average $r_{\text {sites }}$ is found to be between 0.05 and 0.20 (see Table 1). 


\section{Data availability statement}

The data that support the findings of this study are available from the authors upon reasonable request.

\section{Author contributions}

J.H., S.C., C.G., T.C.T.M., E.S., S.L., C.M.D., T.P.J.K., and M.V. designed research. J.H., S.C., C.G., F.S.R., M.S., I.I., performed research. J.H., S.C., C.G., F.S.R., I.I., J.R.K., E.S., S.L., C.M.D., T.P.J.K., and M.V. contributed reagents/analytic tools. J.H., S.C., C.G., T.C.T.M., M.M.J.B., F.S.R., E.S., S.L., C.M.D., T.P.J.K., and M.V. analysed the data. All authors discussed the results and contributed to the writing of the paper. J.H., S.C., and C.G. contributed equally to this work. Correspondence and requests for materials should be addressed to M.V.

\section{Conflict of interest}

The authors declare no conflict of interest.

\section{Acknowledgments}

We acknowledge support from the Centre for Misfolding Diseases (J.H., S.C., T.C.T.M., M.M.J.B., F.S.R., M.S., J.R.K., C.M.D., T.P.J.K., and M.V.); the Agency for Science, Technology and Research, Singapore (S.C.); the NIH-Oxford/Cambridge Scholars Program (M.M.J.B.); the Cambridge Commonwealth, European and International Trust (M.M.J.B.); the Frances and Augustus Newman Foundation (T.P.J.K.); the UK Biotechnology and Biochemical Sciences Research Council (C.M.D. and M.V.); and the Wellcome Trust (C.M.D., T.P.J.K., and M.V.). This work was supported by the Intramural Research Program of the National Institute of Diabetes and Kidney Diseases, NIH. 


\section{Figure Captions}

Figure 1. Schematic illustration of the strategy used in the present work. $A \beta 42$ aggregation kinetics were performed in the absence and the presence of DMPC lipid vesicles that were either free or contain different concentrations of cholesterol. Subsequently, the effect of the lipid vesicles was assessed at a microscopic step level.

Figure 2: DMPC:cholesterol vesicles accelerate $A \beta 42$ aggregation. (a) Comparison of the kinetic profiles for the aggregation of a $2 \mu \mathrm{M} A \beta 42$ sample in the presence of either DMPC vesicles or DMPC:cholesterol vesicles containing increasing concentrations of cholesterol up to $15 \%$ at a $[\mathrm{L}] /[\mathrm{P}]$ ratio of 50 . (b) Evolution of normalised $t_{1 / 2}$ of the aggregation kinetics of $A \beta 42$ against $[\mathrm{L}] /[\mathrm{P}]$ as derived from the data in Supplementary Fig. 1. (c) Evolution of normalised $t_{1 / 2}$ of the aggregation kinetics of $A \beta 42$ against the percentage of cholesterol within the membrane as derived from the data in Supplementary Figs. 1-3. Note the change of the DMPC membrane properties from a single liquid disordered phase $\left(l_{d}\right)$ to a liquid ordered phase $\left(I_{0}\right)$ in the presence of increasing concentrations of cholesterol. Throughout, error bars indicate standard deviation among independent replicates.

Figure 3. Biophysical characterisation of the effects of DMPC:cholesterol vesicles on $A \beta 42$ fibrils. (a) AFM images of ThT-free $A \beta 42$ samples at time $0 \mathrm{~h}$ and after $12 \mathrm{~h}$ incubation at $37^{\circ} \mathrm{C}$ in the absence or the presence of DMPC:cholesterol vesicles containing 0 or $15 \%$ cholesterol at $[\mathrm{L}] /[\mathrm{P}]$ ratio of 10 . Similar height distributions are observed for A $\beta 42$ fibrils irrespective whether they are formed in the absence or the presence of lipid vesicles (see red and blue marks on the images). (b-e) Cryo-EM images of ThT-free A $\beta 42$ fibrils. (b,c) are two representative images of A $\beta 42$ fibrils formed in the absence of DMPC:cholesterol vesicles containing $15 \%$ cholesterol at $[L] /[P]$ ratio of 10 , while $(d, e)$ are two representative images formed in their presence. White arrows indicate the presence of the DMPC:cholesterol vesicles. (f) Second derivative of the IR amplitude of $A \beta 42$ fibrils formed in the absence or the presence of DMPC:cholesterol vesicles containing 0 or $15 \%$ cholesterol at $[\mathrm{L}] /[\mathrm{P}]$ ratio of 10 . 
Figure 4. DMPC:cholesterol vesicles accelerate $A \beta 42$ primary nucleation by up to 20-fold through a heterogeneous nucleation process. (a) Comparison of normalised $t_{\text {lag }}$ and $t_{\text {growth }}$ derived from the aggregation profiles of $2 \mu \mathrm{M} A \beta 42$ in the presence of increasing concentrations of DMPC:cholesterol vesicles containing $10 \%$ cholesterol as a function of the $[\mathrm{L}] /[\mathrm{P}]$ ratio (see Supplementary Fig. 1). (b) Halftime, $t_{1 / 2}$, of $A \beta 42$ aggregation as a function of the initial $A \beta 42$ monomer concentration in the absence or presence of DMPC:cholesterol vesicles containing $10 \%$ cholesterol at $[\mathrm{L}] /[\mathrm{P}]$ ratios of 50 and 200. (c) Kinetic profiles of the aggregation reaction of $1.2 \mu \mathrm{M} \mathrm{A} \beta 42$ in the presence of one molar equivalent of Brichos $(1.2 \mu \mathrm{M})$, and DMPC or DMPC:cholesterol vesicles containing $10 \%$ cholesterol at $[\mathrm{L}] /[\mathrm{P}]$ ratios of 50 and 100. (d) Aggregation profiles of $2 \mu \mathrm{M} \mathrm{A} \beta 42$ in the absence and presence of DMPC:cholesterol vesicles containing 10\% cholesterol at increasing $[\mathrm{L}] /[\mathrm{P}]$ ratios. The solid lines show predictions for the resulting reaction profiles when the rate constants of the primary and secondary pathways are modified to include the lipidinduced nucleation process. (e) Evolution of apparent reaction rate constants with increasing $[\mathrm{L}] /[\mathrm{P}]$ ratios of DMPC:cholesterol vesicles containing $10 \%$ cholesterol. $\mathrm{k}^{\text {lipids }} / \mathrm{k}$ represents in each case either $k_{+}{ }^{\prime} k_{\mathrm{n}}{ }^{\prime} / k_{+} k_{\mathrm{n}}$ or $k_{+}{ }^{\prime} k_{2}{ }^{\prime} / k_{+} k_{2}$, where $k_{\mathrm{n}}{ }^{\prime}, k_{+}{ }^{\prime}$, and $k_{2}{ }^{\prime}$ are the apparent rate constants of primary nucleation, elongation, and secondary nucleation, respectively, in the presence of vesicles. Note the significant increase in $k_{+}{ }^{\prime} k_{n}{ }^{\prime}$ as compared to $k_{+}{ }^{\prime} k_{2}{ }^{\prime}$ as the $[\mathrm{L}] /[\mathrm{P}]$ increases (in the yellow zone). (f) Numerical calculations of the reaction profiles for a solution of $2 \mu \mathrm{M} A \beta 42$ with (blue) and without (black) an increase by 20 -fold the rate constant of primary nucleation, $k_{\mathrm{n}}$ ' (see Material and Methods). Inset shows $t_{1 / 2}$ of calculated and experimental curves showing a maximal effect of lipids. Throughout, error bars indicate standard deviation among independent replicates.

Figure 5. DMPC:cholesterol vesicles catalyse the formation of $A \beta 42$ oligomers through heterogeneous nucleation. (a) Log-log plot of $\alpha m(0)^{n_{c}}$ as a function of the initial concentration of A 442 monomers, $m(0)$. (b) Log-log plot of $\alpha$, the slope of acceleration $\varepsilon$ of primary nucleation, as a function of $\theta$, the surface coverage by cholesterol (\% of cholesterol within the membrane). A schematic representation of A $\beta 42$ heterogeneous primary nucleation on the surface of DMPC:cholesterol vesicles is also shown. Note that heterogeneous primary nucleation occurs as a result of the 
cooperativity in the interaction of several cholesterol molecules with $A \beta 42$. (c) Ratio of the apparent primary nucleation rate constants in the absence (black) and in the presence (green) of DMPC:cholesterol vesicles containing $10 \%$ cholesterol at a $[\mathrm{L}] /[\mathrm{P}]$ ratio of 50 and either in the presence or the absence of 3 molar equivalents of bexarotene to $A \beta 42 . k_{n}$ is the rate constant of homogeneous primary nucleation and $k_{n}$ ' is the sum of the rate constants of both, homogeneous and heterogeneous primary nucleation, $\mathrm{K}^{\mathrm{app}} / \mathrm{K}$ represents either $k_{\mathrm{n}}{ }^{\text {app }} / k_{\mathrm{n}}$ or $k_{\mathrm{n}}{ }^{\text {app }} / k_{\mathrm{n}}$. (d) Acceleration in the formation of the total number of oligomers generated through increasing rates of primary nucleation. Error bars in (a) and (b) were derived from the extrapolation of the data from Supplementary Figs. 1-3 and 13, respectively. Error bars in (c) indicate standard deviation between independent replicates.

Figure 6. DMPC:cholesterol vesicles introduces a heterogeneous nucleation pathway in the $A \beta 42$ aggregation process. We present a scheme showing the proposed model of $A \beta 42$ aggregation in the presence of catalytic sites formed by DMPC:cholesterol membrane. In the presence of DMPC:cholesterol vesicles, the primary nucleation of $A \beta 42$ occurs through either homogeneous nucleation, in which A $\beta 42$ nuclei are formed solely from the interactions of $A \beta 42$ monomers, or through heterogeneous nucleation, in which $A \beta 42$ nuclei are formed from the interactions of A $\beta 42$ monomers and lipid membranes. In either type of primary nucleation, once a critical number of fibrils are formed, secondary nucleation becomes the major source for generating new oligomers. 


\begin{tabular}{|c|c|c|}
\hline$\theta$ & {$[\mathrm{L}] /[\mathrm{P}]$ at maximal effect } & $\mathrm{r}_{\text {sites }}$ \\
\hline 0.05 & 400 & 0.05 \\
\hline 0.10 & 200 & 0.20 \\
\hline 0.15 & 20 & 0.07 \\
\hline 0.20 & 10 & 0.08 \\
\hline
\end{tabular}

Table 1: Estimation of the number of accessible nucleation sites, $r_{\text {sites }}$, per $\mathrm{A} \beta_{42}$ monomer for given monomer to lipid concentration ratios. $\theta$ is the surface coverage by cholesterol. 


\section{References}

1. Alzheimer's Association. 2012 Alzheimer's disease facts and figures. Alzheimer's Dement. 8, 131-168 (2012).

2. Dobson, C. M. Protein folding and misfolding. Nature 426, 884-890 (2003).

3. Haass, C. \& Selkoe, D. J. Soluble protein oligomers in neurodegeneration: lessons from the Alzheimer's amyloid beta-peptide. Nat. Rev. Mol. Cell Biol. 8, 101-112 (2007).

4. Knowles, T. P. J., Vendruscolo, M. \& Dobson, C. M. The amyloid state and its association with protein misfolding diseases. Nat. Rev. Mol. Cell Biol. 15, 384396 (2014).

5. Tanzi, R. E. \& Bertram, L. Twenty years of the Alzheimer's disease amyloid hypothesis: A genetic perspective. Cell 120, 545-555 (2005).

6. Necula, M., Kayed, R., Milton, S. \& Glabe, C. G. Small molecule inhibitors of aggregation indicate that amyloid $\beta$ oligomerization and fibrillization pathways are independent and distinct. J. Biol. Chem. 282, 10311-10324 (2007).

7. Lansbury, P. T. \& Lashuel, H. A. A century-old debate on protein aggregation and neurodegeneration enters the clinic. Nature 443, 774-779 (2006).

8. Galvagnion, C. et al. Lipid vesicles trigger $\alpha$-synuclein aggregation by stimulating primary nucleation. Nat. Chem. Biol. 11, 229-234 (2015).

9. Di Paolo, G. \& Kim, T.-W. Linking lipids to Alzheimer's disease: cholesterol and beyond. Nat. Rev. Neurosci. 12, 284-296 (2011).

10. Gellermann, G. P. et al. Raft lipids as common components of human extracellular amyloid fibrils. Proc. Natl. Acad. Sci. U. S. A. 102, 6297-6302 (2005).

11. Bertram, L. \& Tanzi, R. E. Thirty years of Alzheimer's disease genetics : the implications of systematic meta-analyses. Nat. Rev. Neurosci. 9, 768-778 (2008).

12. Corder, E. H. et al. Gene dose of apolipoprotein E type 4 allele and the risk of 
Alzheimer's disease in late onset families. Science 261, 921-923 (1993).

13. Bu, G. Apolipoprotein $\mathrm{E}$ and its receptors in Alzheimer's disease: pathways, pathogenesis and therapy. Nat. Rev. Neurosci. 10, 333-344 (2009).

14. Holtzman, D. M. Role of apoE/Aß interactions in the pathogenesis of Alzheimer's disease and cerebral amyloid angiopathy. J. Mol. Neurosci. 17, 147-155 (2001).

15. Popp, J. et al. Cerebral and extracerebral cholesterol metabolism and CSF markers of Alzheimer's disease. Biochem. Pharmacol. 86, 37-42 (2013).

16. Mori, T. et al. Cholesterol accumulates in senile plaques of Alzheimer disease patients and in transgenic APP(SW) mice. J. Neuropathol. Exp. Neurol. 60, 778-785 (2001).

17. Zissimopoulos, J. M. et al. Sex and Race Differences in the Association Between Statin Use and the Incidence of Alzheimer Disease. JAMA Neurol. 111, 390-400 (2016).

18. Dietschy, J. M. \& Turley, S. D. Cholesterol metabolism in the brain. Curr. Opin. Lipidol. 12, 105-112 (2001).

19. Vance, J. E. Dysregulation of cholesterol balance in the brain: contribution to neurodegenerative diseases. Dis. Model. Mech. 5, 746-755 (2012).

20. Wood, W. G., Li, L., Müller, W. E. \& Eckert, G. P. Cholesterol as a causitive agent in Alzheimer disease a debatable hypothesis. J. Neurochem. 129, 559572 (2014).

21. Di Scala, C., Chahinian, H., Yahi, N., Garmy, N. \& Fantini, J. Interaction of Alzheimer's $\beta$-amyloid peptides with cholesterol: mechanistic insights into amyloid pore formation. Biochemistry 53, 4489-4502 (2014).

22. Ghribi, O., Larsen, B., Schrag, M. \& Herman, M. M. High cholesterol content in neurons increases BACE, $\beta$-amyloid, and phosphorylated tau levels in rabbit hippocampus. Exp. Neurol. 200, 460-467 (2006).

23. Barrett, P. J. et al. The amyloid precursor protein has a flexible transmembrane 
domain and binds cholesterol. Science 117, 2010-2013 (2012).

24. Evangelisti, E. et al. Membrane lipid composition and its physicochemical properties define cell vulnerability to aberrant protein oligomers. J. Cell Sci. 125, 2416-2427 (2012).

25. Hellstrand, E., Sparr, E. \& Linse, S. Retardation of $A \beta$ fibril formation by phospholipid vesicles depends on membrane phase behavior. Biophys. J. 98, 2206-2214 (2010).

26. Yip, C. M., Elton, E. a, Darabie, a a, Morrison, M. R. \& McLaurin, J. Cholesterol, a modulator of membrane-associated $\mathrm{A}$ beta- fibrillogenesis and neurotoxicity. J. Mol. Biol. 311, 723-734 (2001).

27. Hellstrand, E., Boland, B., Walsh, D. M. \& Linse, S. Amyloid $\beta$-protein aggregation produces highly reproducible kinetic data and occurs by a twophase process. ACS Chem. Neurosci. 1, 13-18 (2010).

28. Knowles, T. P. J. et al. An analytical solution to the kinetics of breakable filament assembly. Science 326, 1533-1537 (2009).

29. Cohen, S. I. A. et al. Proliferation of amyloid- $\beta 42$ aggregates occurs through a secondary nucleation mechanism. Proc. Natl. Acad. Sci. U. S. A. 110, 97589763 (2013).

30. Cohen, S. I. A., Vendruscolo, M., Dobson, C. M. \& Knowles, T. P. J. From macroscopic measurements to microscopic mechanisms of protein aggregation. J. Mol. Biol. 421, 160-171 (2012).

31. Meisl, G. et al. Molecular mechanisms of protein aggregation from global fitting of kinetic models. Nat. Protoc. 11, 252-272 (2016).

32. Meisl, G., Yang, X., Frohm, B., Knowles, T. P. J. \& Linse, S. Quantitative analysis of intrinsic and extrinsic factors in the aggregation mechanism of Alzheimer-associated Aß-peptide. Sci. Rep. 6, 18728 (2016).

33. Meisl, G. et al. Differences in nucleation behavior underlie the contrasting aggregation kinetics of the $A \beta 40$ and $A \beta 42$ peptides. Proc. Natl. Acad. Sci. U. S. A. 111, 9384-9389 (2014). 
34. Michaels, T. C. T., Lazell, H. W., Arosio, P. \& Knowles, T. P. J. Dynamics of protein aggregation and oligomer formation governed by secondary nucleation. J. Chem. Phys. 143, 54901 (2015).

35. Arosio, P., Vendruscolo, M., Dobson, C. M. \& Knowles, T. P. J. Chemical kinetics for drug discovery to combat protein aggregation diseases. Trends Pharmacol. Sci. 35, 127-135 (2014).

36. Arosio, P. et al. Kinetic analysis reveals the diversity of microscopic mechanisms through which molecular chaperones suppress amyloid formation. Nat. Commun. 7, 10948 (2016).

37. Cohen, S. I. A. et al. A molecular chaperone breaks the catalytic cycle that generates toxic A $\beta$ oligomers. Nat. Struct. Mol. Biol. 22, 207-213 (2015).

38. Aprile, F. a., Sormanni, P. \& Vendruscolo, M. A rational design strategy for the selective activity enhancement of a molecular chaperone toward a target substrate. Biochemistry 54, 5103-5112 (2015).

39. Sormanni, P., Aprile, F. A. \& Vendruscolo, M. Rational design of antibodies targeting specific epitopes within intrinsically disordered proteins. Proc. Natl. Acad. Sci. U. S. A. 112, 9902-9907 (2015).

40. Heller, G. T., Sormanni, P. \& Vendruscolo, M. Targeting disordered proteins with small molecules using entropy. Trends Biochem. Sci. 40, 491-496 (2015).

41. Habchi, J. et al. An anti-cancer drug suppresses the primary nucleation reaction that initiates the formation of toxic $A \beta$ aggregates associated with Alzheimer's disease. Sci. Adv. 2, e1501244 (2016).

42. Habchi, J. et al. Systematic development of small molecules to inhibit specific microscopic steps of A 342 aggregation in Alzheimer's disease. Proc. Natl. Acad. Sci. 114, E200-E208 (2016).

43. Sastry, P. S. Lipids of nervous tissue: Composition and metabolism. Prog. Lipid Res. 24, 69-176 (1985).

44. van Meer, G., Voelker, D. R. \& Feigenson, G. W. Membrane lipids: where they are and how they behave. Nat. Rev. Mol. Cell Biol. 9, 112-124 (2008). 
45. Mouritsen, O. G. Life-as a matter of fat. (Springer, 2005).

46. Cotman, C. W., Blank, M., Moehl, a. \& Snyder, F. Lipid composition of synaptic plasma membranes isolated from rat brain by zonal centrifugation. Biochemistry 8, 4606-4612 (1969).

47. Nagle, J. F. et al. X-ray structure determination of fully hydrated $L$ alpha phase dipalmitoylphosphatidylcholine bilayers. Biophys. J. 70, 1419-1431 (1996).

48. Kucerka, N. et al. Structure of fully hydrated fluid phase DMPC and DLPC lipid bilayers using X-ray scattering from oriented multilamellar arrays and from unilamellar vesicles. Biophys. J. 88, 2626-2637 (2005).

49. Chi, E. Y. et al. Lipid membrane templates the ordering and induces the fibrillogenesis of Alzheimer's disease amyloid- $\beta$ peptide. Proteins Struct. Funct. Genet. 72, 1-24 (2008).

50. Niu, Z. et al. The molecular structure of Alzheimer $\beta$-amyloid fibrils formed in the presence of phospholipid vesicles. Angew. Chemie - Int. Ed. 53, 92949297 (2014).

51. Simons, K. \& Vaz, W. L. C. Model systems, lipid rafts, and cell membranes. Annu. Rev. Biophys. Biomol. Struct. 33, 269-295 (2004).

52. Filippov, A., Orädd, G. \& Lindblom, G. The effect of cholesterol on the lateral diffusion of phospholipids in oriented bilayers. Biophys. J. 84, 3079-3086 (2003).

53. Barrett, M. A. et al. Solubility of cholesterol in lipid membranes and the formation of immiscible cholesterol plaques at high cholesterol concentrations. Soft Matter 9, 9342-9351 (2013).

54. Almeida, P. F., Vaz, W. L. \& Thompson, T. E. Lateral diffusion in the liquid phases of dimyristoylphosphatidylcholine/cholesterol lipid bilayers: a free volume analysis. Biochemistry 31, 6739-6747 (1992).

55. Blume, A. A comparative study of the phase transitions of phospholipid bilayers and monolayers. Biochim. Biophys. Acta - Biomembr. 557, 32-44 (1979). 
56. Wimley, W. C. \& Thompson, T. E. Transbilayer and interbilayer phospholipid exchange in

dimyristoylphosphatidylcholine/dimyristoylphosphatidylethanolamine large unilamellar vesicles. Biochemistry 30, 1702-1709 (1991).

57. Harris, F. M., Best, K. B. \& Bell, J. D. Use of laurdan fluorescence intensity and polarization to distinguish between changes in membrane fluidity and phospholipid order. Biochim. Biophys. Acta - Biomembr. 1565, 123-128 (2002).

58. Aguilar, L. F. et al. Differential dynamic and structural behavior of lipidcholesterol domains in model membranes. PLoS One 7, e40254 (2012).

59. Galvagnion, C. et al. Chemical properties of lipids strongly affect the kinetics of the membrane-induced aggregation of a-synuclein. Proc. Natl. Acad. Sci. U. S. A. 113, 7065-70 (2016).

60. De Meyer, F. \& Smit, B. Effect of cholesterol on the structure of a phospholipid bilayer. Proc. Natl. Acad. Sci. U. S. A. 106, 3654-3658 (2009).

61. de Jongh, H. H. J., Goormaghtigh, E. \& Killian, J. A. Analysis of circular dichroism spectra of oriented protein-lipid complexes: Toward a general application. Biochemistry 33, 14521-14528 (1994).

62. Linse, S. \& Lund, M. Surface Effects on Aggregation Kinetics of Amyloidogenic Peptides. J. Am. Chem. Soc. 136, 11555-11850 (2014).

63. Ruggeri, F. S. et al. Nanoscale studies link amyloid maturity with polyglutamine diseases onset. Sci. Rep. 6, 31155 (2016).

64. Zandomeneghi, G., Krebs, M. R. H., McCammon, M. G. \& Fändrich, M. FTIR reveals structural differences between native beta-sheet proteins and amyloid fibrils. Protein Sci. 13, 3314-21 (2004).

65. Arosio, P., Knowles, T. P. J. \& Linse, S. On the lag phase in amyloid fibril formation. Phys. Chem. Chem. Phys. 17, 7606-7618 (2015).

66. Cohen, S. I. A., Vendruscolo, M., Dobson, C. M. \& Knowles, T. P. J. Nucleated polymerization with secondary pathways. II. Determination of self-consistent 
solutions to growth processes described by non-linear master equations. J. Chem. Phys. 135, 65106 (2011).

67. Cohen, S. I. A., Vendruscolo, M., Dobson, C. M. \& Knowles, T. P. J. Nucleated polymerization with secondary pathways. III. Equilibrium behavior and oligomer populations. J. Chem. Phys. 135, 65107 (2011).

68. Cohen, S. I. A. et al. Nucleated polymerization with secondary pathways. I. Time evolution of the principal moments. J. Chem. Phys. 135, 65105 (2011).

69. Simons, M. et al. Cholesterol depletion inhibits the generation of $\beta$-amyloid in hippocampal neurons. Proc. Natl. Acad. Sci. U. S. A. 95, 6460-6464 (1998).

70. Hong, S. et al. Soluble $A \beta$ oligomers are rapidly sequestered from brain ISF in vivo and bind GM1 ganglioside on cellular membranes. Neuron 82, 308-319 (2014). 
Chemical kinetics in the absence and presence of lipid vesicles

Primary Nucleation

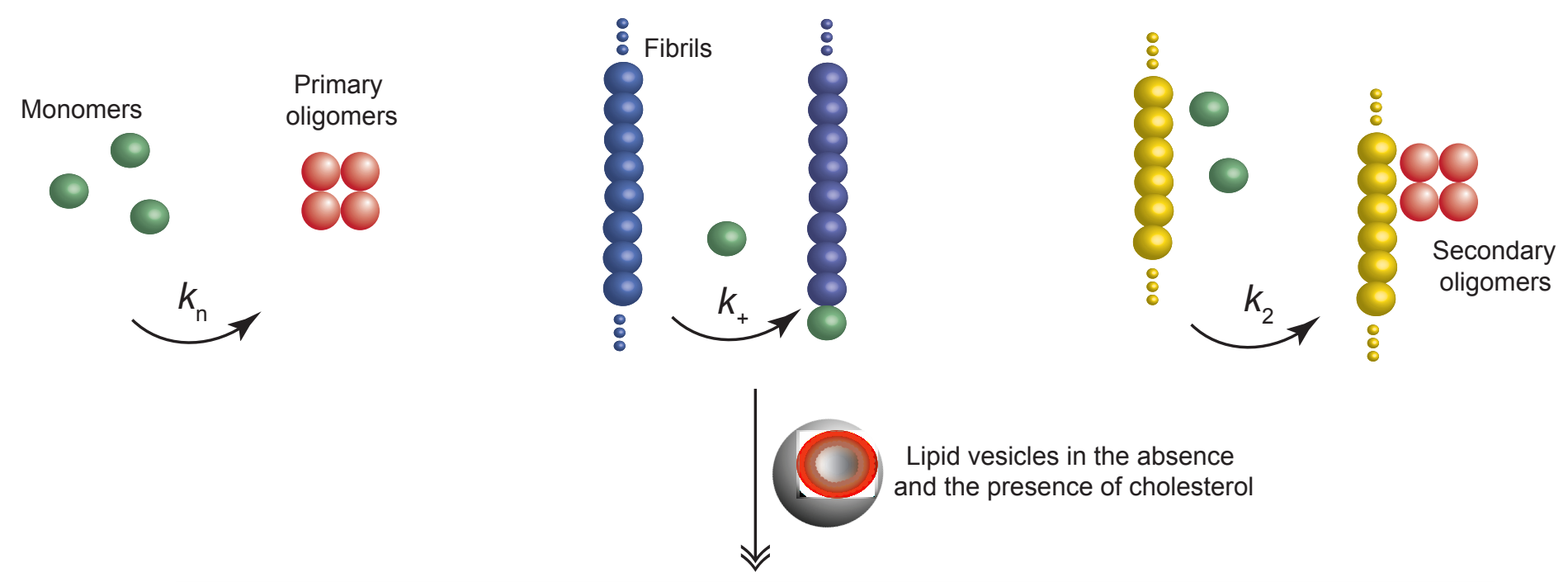

Quantititative description of the role of lipid vesicles in $A \beta 42$ aggregation

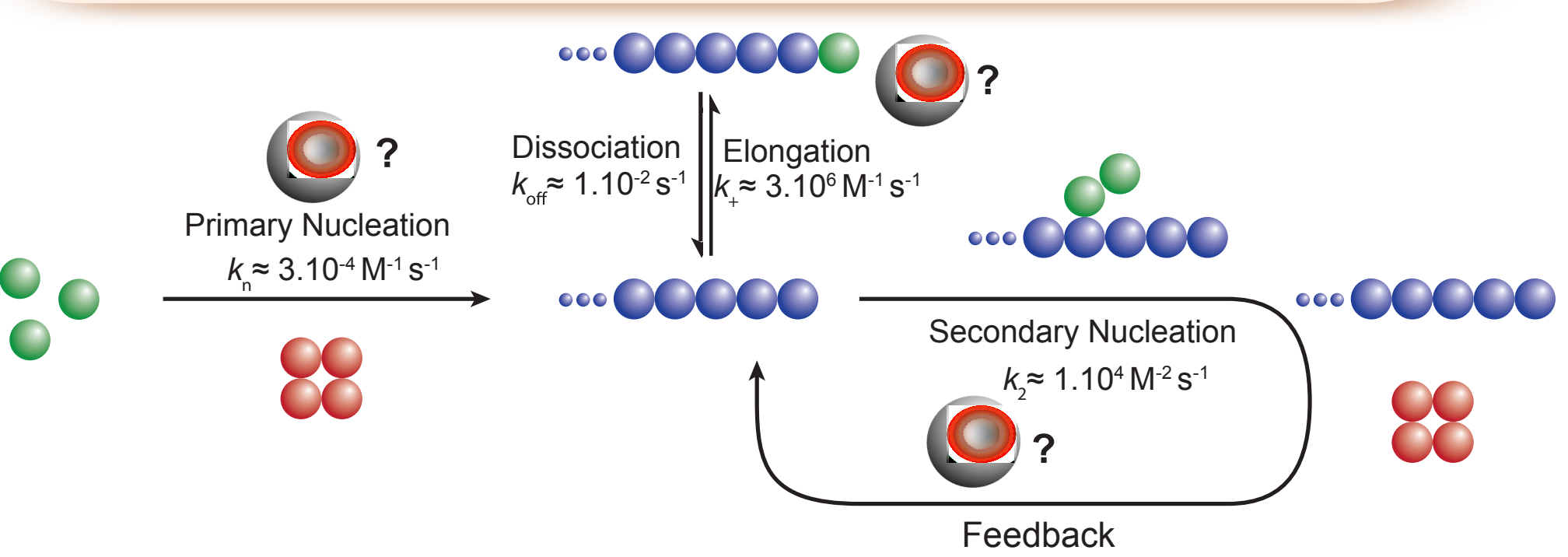


$a$

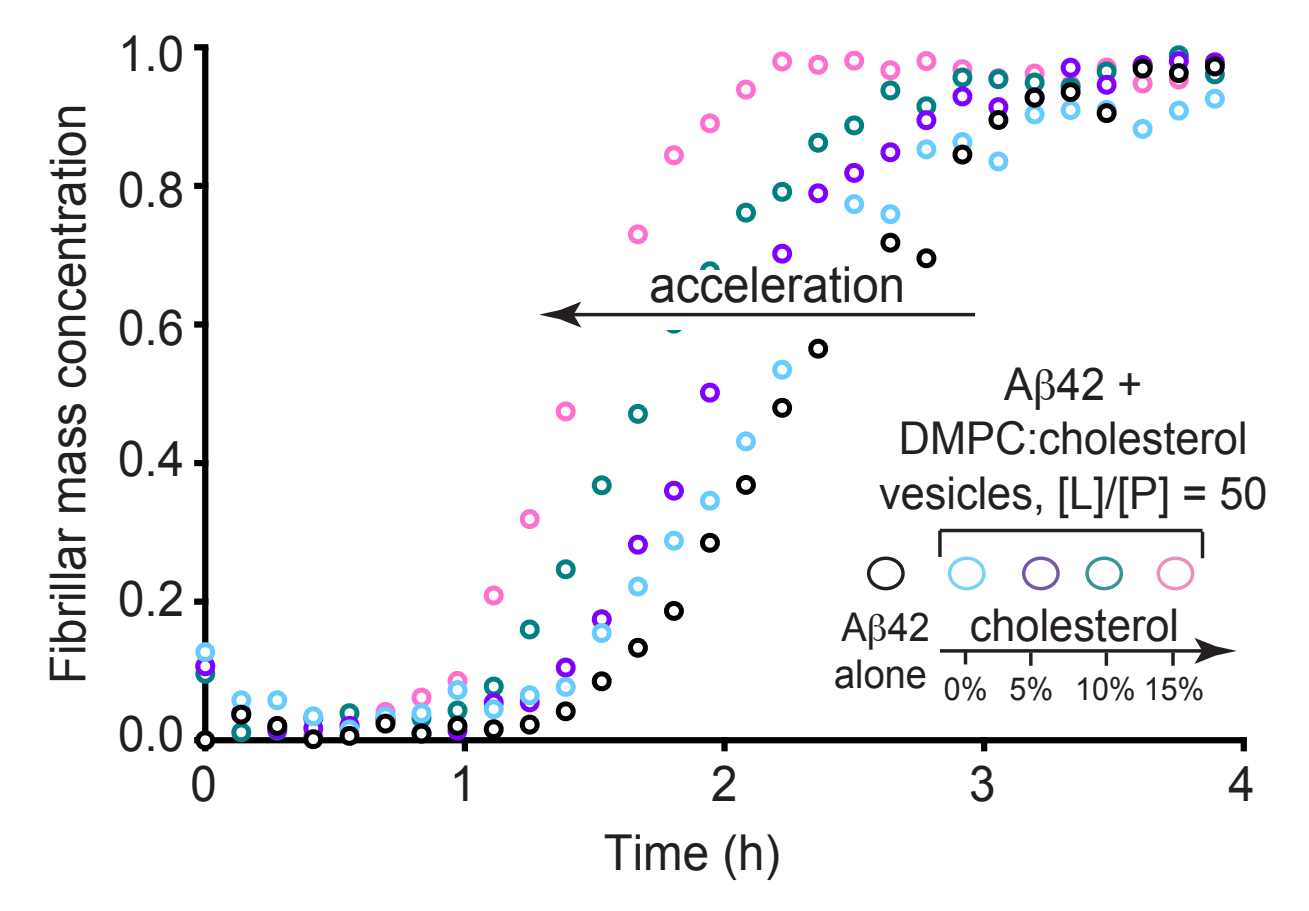

b

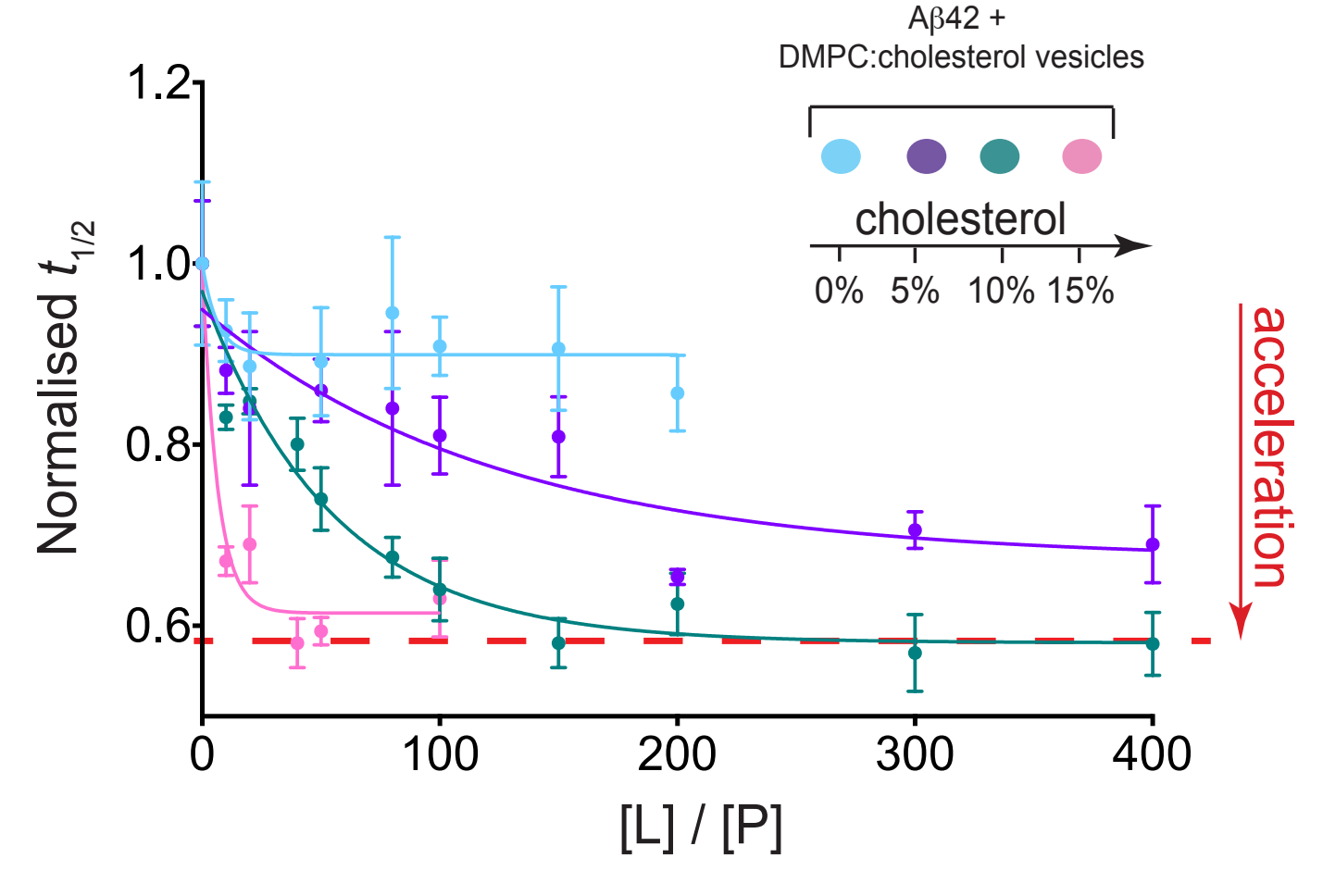

\section{C}

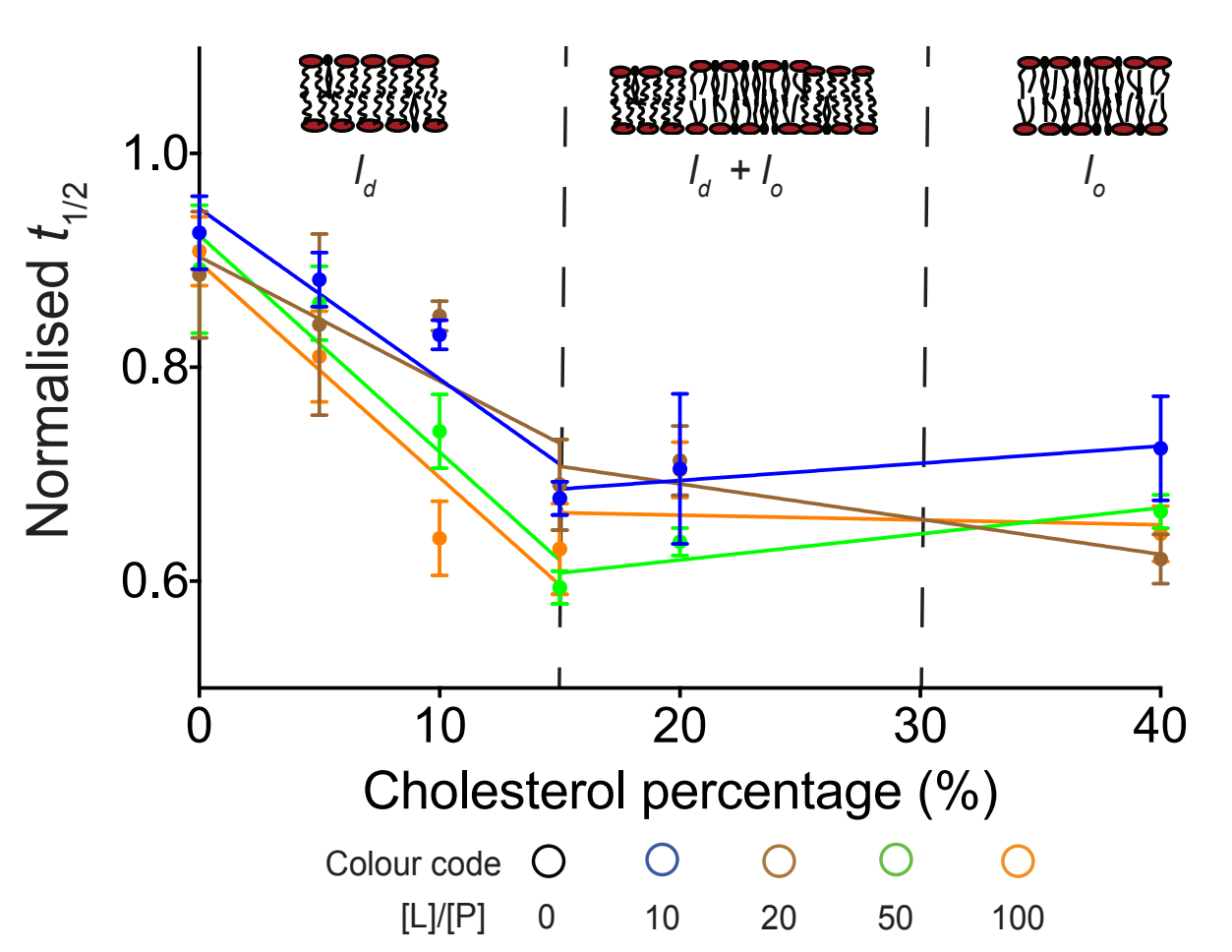




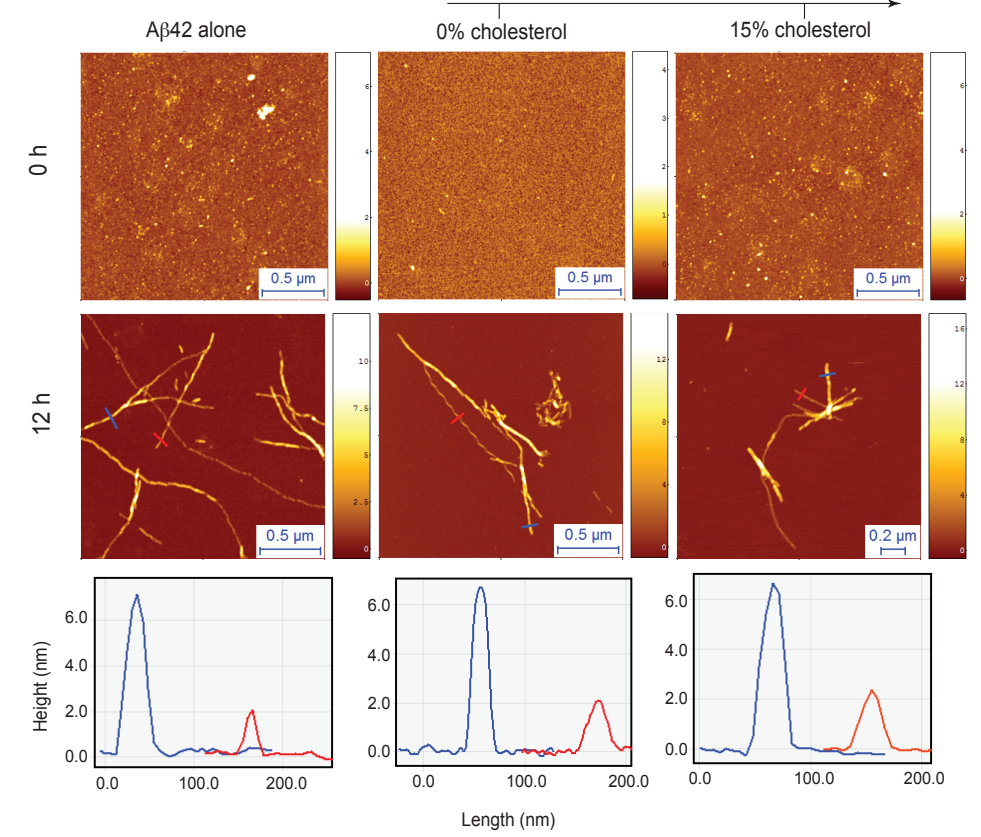

A $\beta 42+$ DMPC:cholesterol vesicles (15\%) at $[\mathrm{L}] /[\mathrm{P}]$ of 10

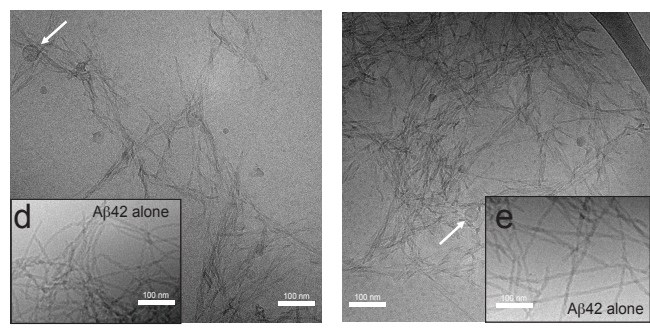

f

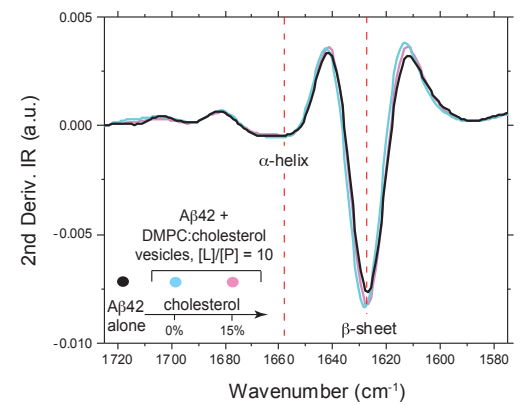


a

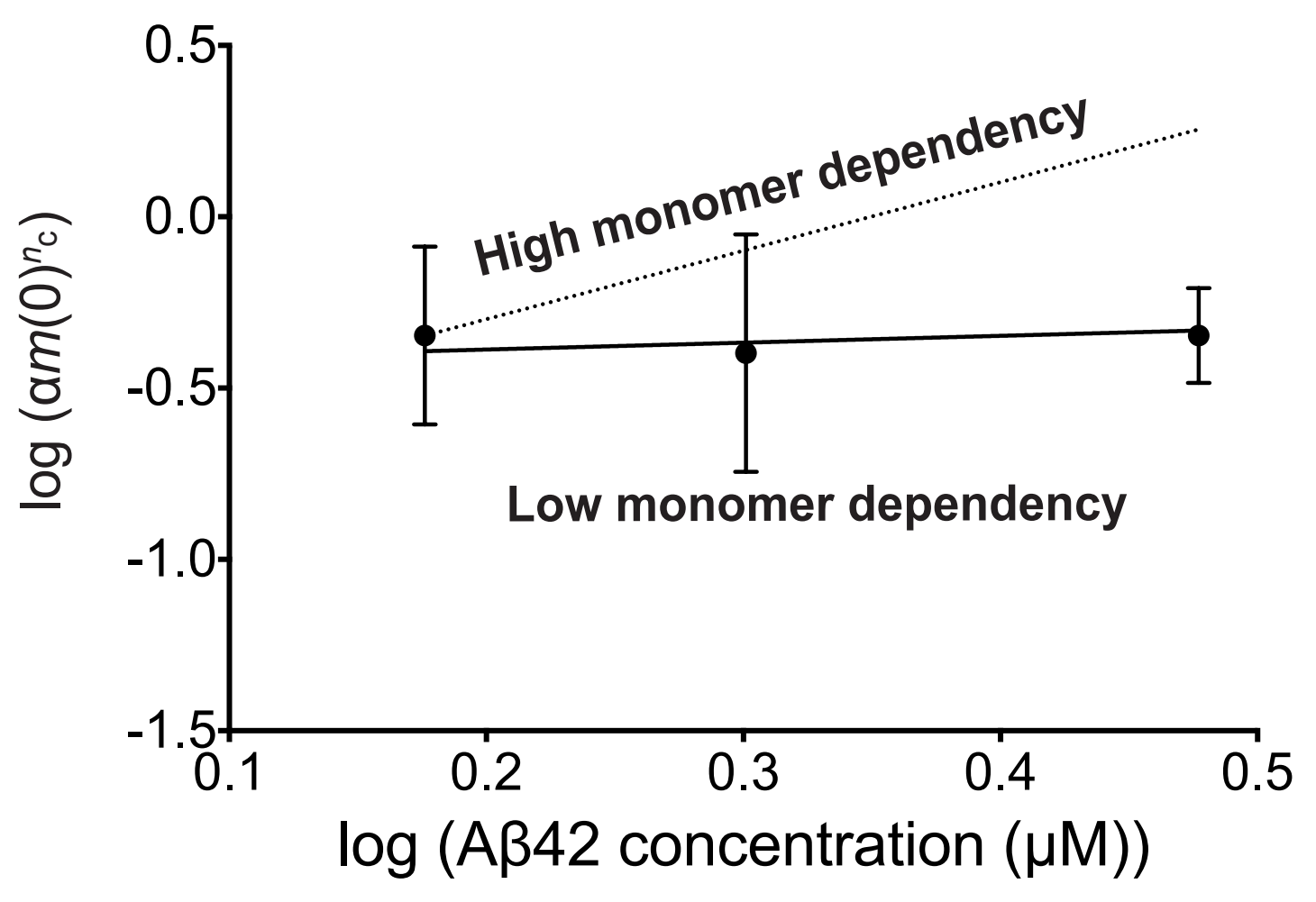

c

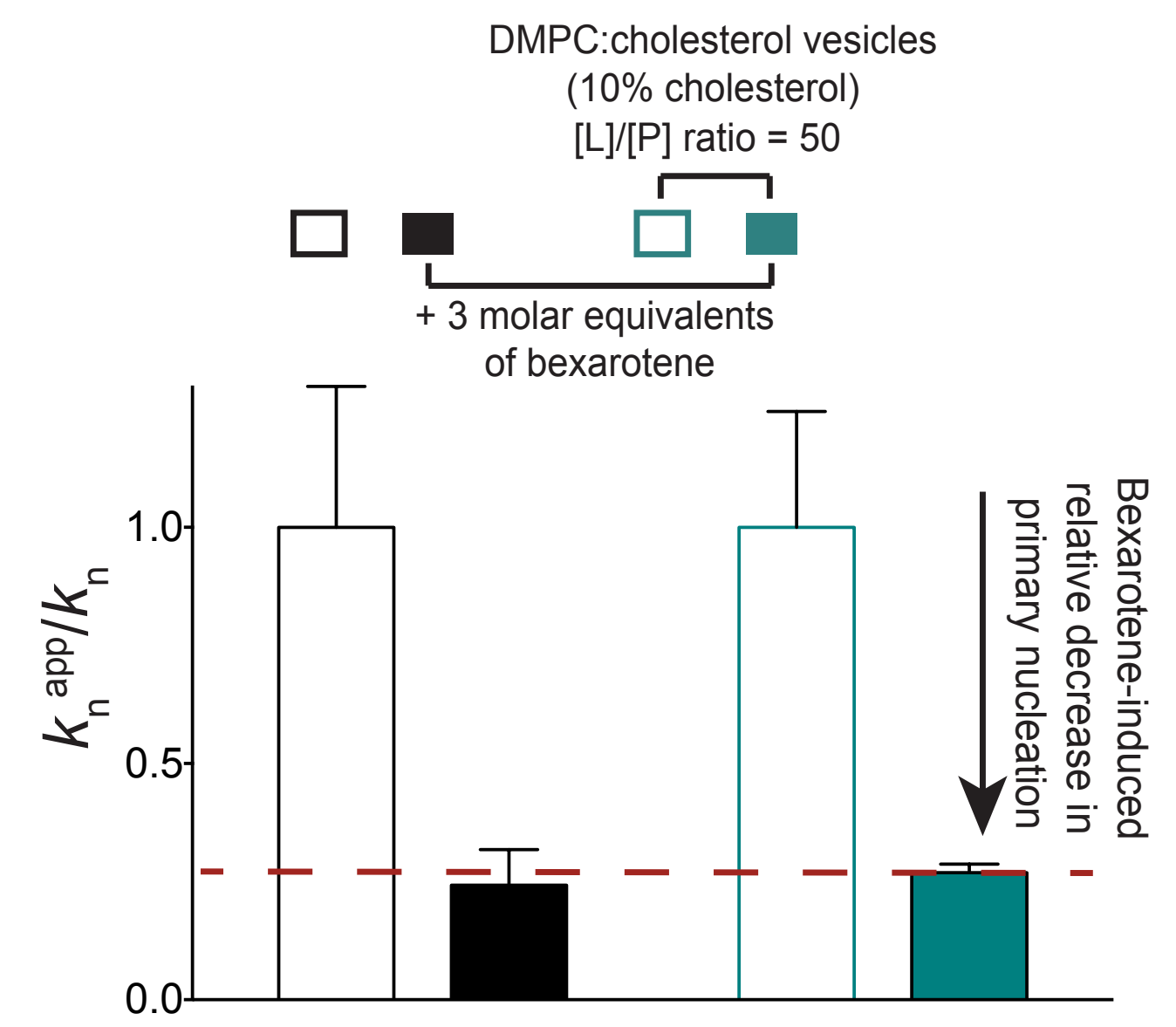

b
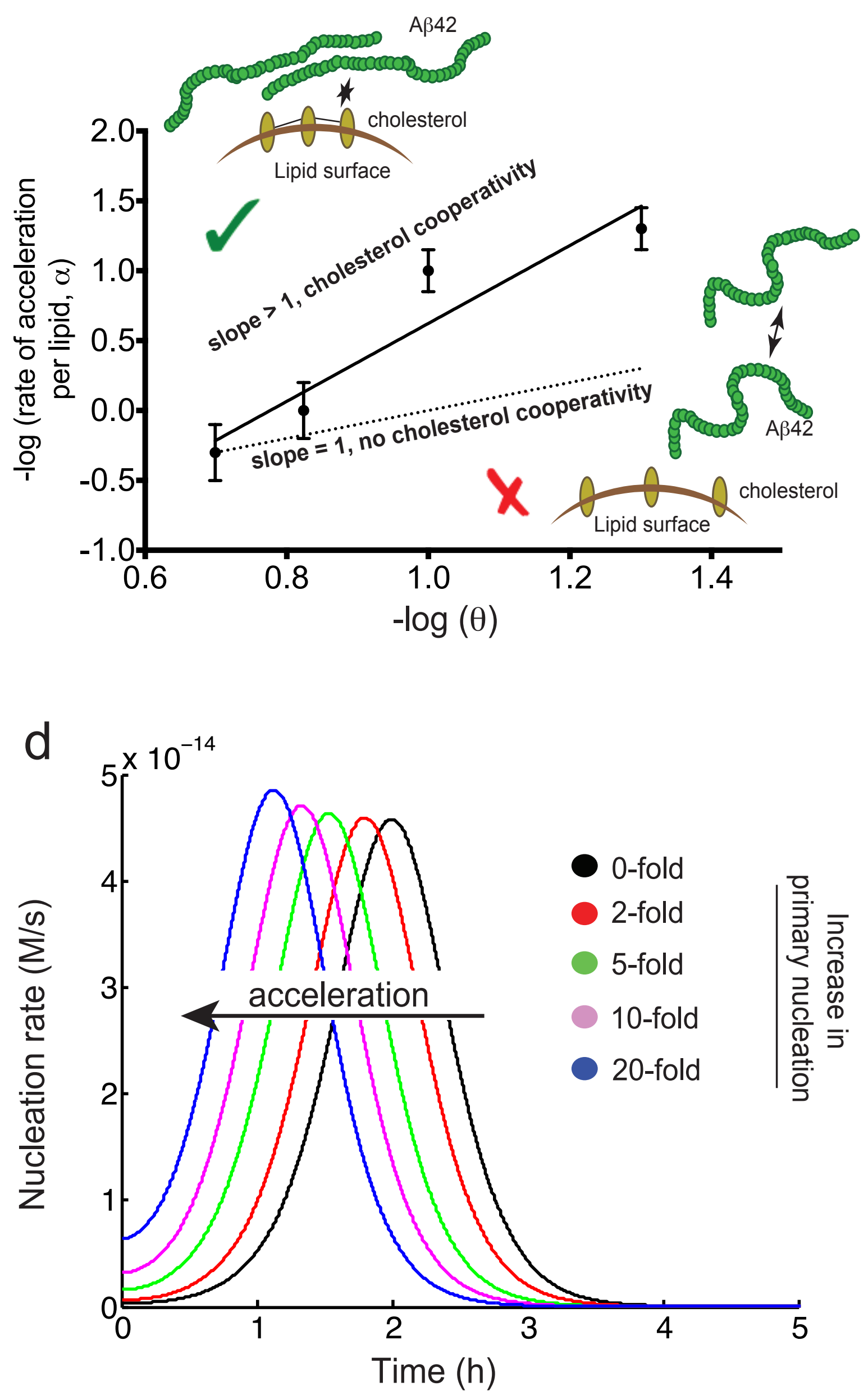
Model of $A \beta 42$ aggregation in the presence of heterogeneous primary nucleation

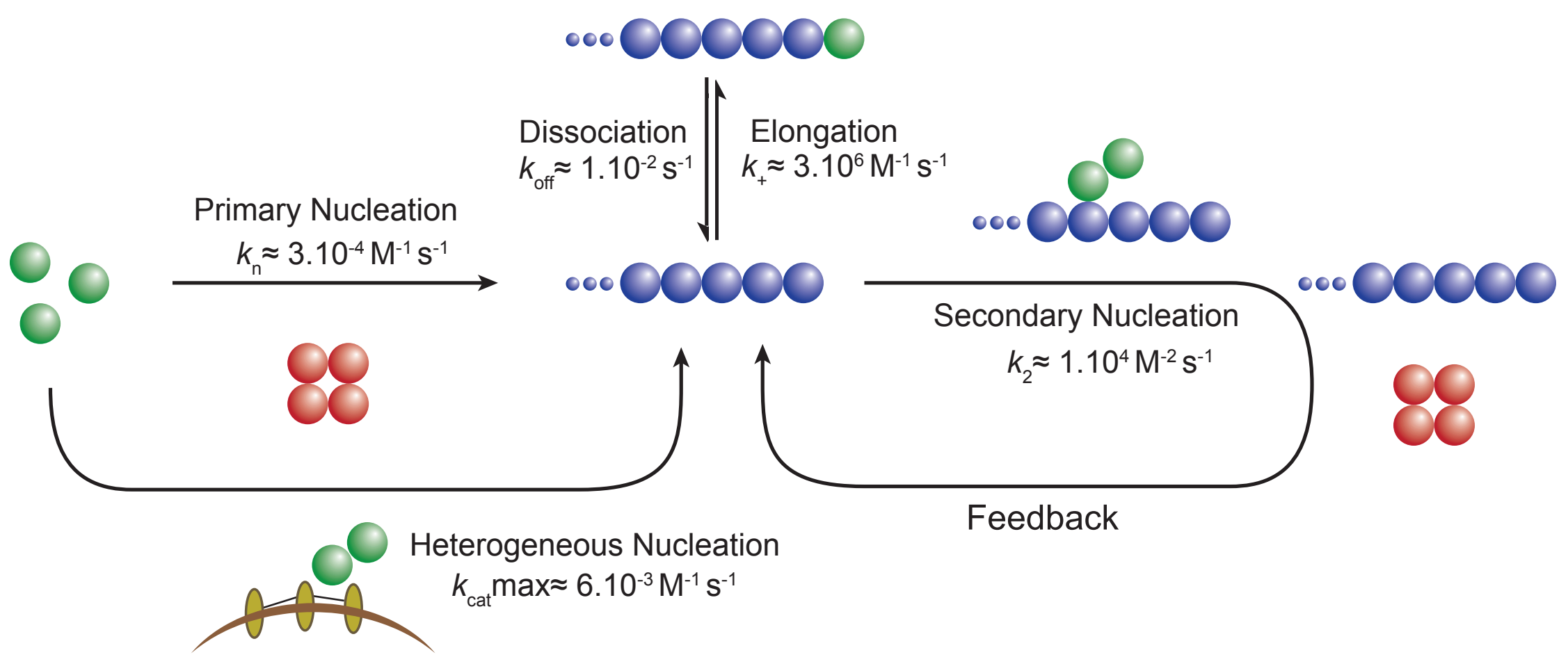

A 342 monomers 88 A $\beta 42$ nuclei ${ }^{\circ} 00000$ Elongating fibrils_Lipid surface 0 Cholesterol 


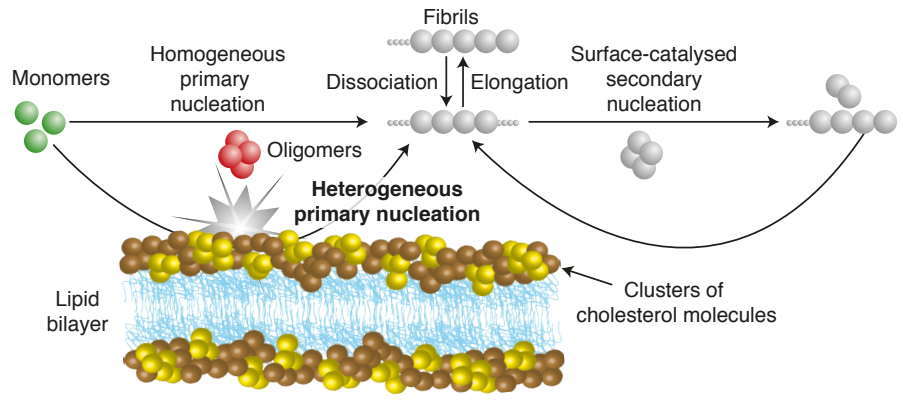

